TRANSACTIONS OF THE

AMERICAN MATHEMATICAL SOCIETY

Volume 359, Number 9, September 2007, Pages 4237-4258

S 0002-9947(07)04247-X

Article electronically published on April 11, 2007

\title{
GRADED ANNIHILATORS OF MODULES OVER THE FROBENIUS SKEW POLYNOMIAL RING, AND TIGHT CLOSURE
}

\author{
RODNEY Y. SHARP
}

\begin{abstract}
This paper is concerned with the tight closure of an ideal $\mathfrak{a}$ in a commutative Noetherian local ring $R$ of prime characteristic $p$. Several authors, including R. Fedder, K-i. Watanabe, K. E. Smith, N. Hara and F. Enescu, have used the natural Frobenius action on the top local cohomology module of such an $R$ to good effect in the study of tight closure, and this paper uses that device. The main part of the paper develops a theory of what are here called 'special annihilator submodules' of a left module over the Frobenius skew polynomial ring associated to $R$; this theory is then applied in the later sections of the paper to the top local cohomology module of $R$ and used to show that, if $R$ is Cohen-Macaulay, then it must have a weak parameter test element, even if it is not excellent.
\end{abstract}

\section{INTRODUCTION}

Throughout the paper, $R$ will denote a commutative Noetherian ring of prime characteristic $p$. We shall always denote by $f: R \longrightarrow R$ the Frobenius homomorphism, for which $f(r)=r^{p}$ for all $r \in R$. Let $\mathfrak{a}$ be an ideal of $R$. The $n$-th Frobenius power $\mathfrak{a}^{\left[p^{n}\right]}$ of $\mathfrak{a}$ is the ideal of $R$ generated by all $p^{n}$-th powers of elements of $\mathfrak{a}$.

We use $R^{\circ}$ to denote the complement in $R$ of the union of the minimal prime ideals of $R$. An element $r \in R$ belongs to the tight closure $\mathfrak{a}^{*}$ of $\mathfrak{a}$ if and only if there exists $c \in R^{\circ}$ such that $c r^{p^{n}} \in \mathfrak{a}^{\left[p^{n}\right]}$ for all $n \gg 0$. We say that $\mathfrak{a}$ is tightly closed precisely when $\mathfrak{a}^{*}=\mathfrak{a}$. The theory of tight closure was invented by M. Hochster and C. Huneke [8, and many applications have been found for the theory; see [10] and [1], for example.

In the case when $R$ is local, several authors have used, as an aid to the study of tight closure, the natural Frobenius action on the top local cohomology module of $R$; see, for example, R. Fedder [4, Fedder and K.-i. Watanabe [5], K. E. Smith [17, N. Hara and Watanabe [6] and F. Enescu [3]. This device is employed in this paper. The natural Frobenius action provides the top local cohomology module of $R$ with a natural structure as a left module over the skew polynomial ring $R[x, f]$ associated

Received by the editors July 8, 2005.

2000 Mathematics Subject Classification. Primary 13A35, 16S36, 13D45, 13E05, 13E10; Secondary $13 \mathrm{H} 10$.

Key words and phrases. Commutative Noetherian ring, prime characteristic, Frobenius homomorphism, tight closure, (weak) test element, (weak) parameter test element, skew polynomial ring, local cohomology, Cohen-Macaulay local ring.

The author was partially supported by the Engineering and Physical Sciences Research Council of the United Kingdom (Overseas Travel Grant Number EP/C538803/1). 
to $R$ and $f$. Sections 1 and 3 develop a theory of what are here called 'special annihilator submodules' of a left $R[x, f]$-module $H$. To explain this concept, we need the definition of the graded annihilator $\operatorname{gr}^{-a n n_{R[x, f]}} H$ of $H$. Now $R[x, f]$ has a natural structure as a graded ring, and gr-ann ${ }_{R[x, f]} H$ is defined to be the largest graded two-sided ideal of $R[x, f]$ that annihilates $H$. On the other hand, for a graded two-sided ideal $\mathfrak{B}$ of $R[x, f]$, the annihilator of $\mathfrak{B}$ in $H$ is defined as

$$
\operatorname{ann}_{H} \mathfrak{B}:=\{h \in H: \theta h=0 \text { for all } \theta \in \mathfrak{B}\} .
$$

I say that an $R[x, f]$-submodule of $H$ is a special annihilator submodule of $H$ if it has the form $\operatorname{ann}_{H} \mathfrak{B}$ for some graded two-sided ideal $\mathfrak{B}$ of $R[x, f]$.

There is a natural bijective inclusion-reversing correspondence between the set of all special annihilator submodules of $H$ and the set of all graded annihilators of submodules of $H$. A large part of this paper is concerned with exploration and exploitation of this correspondence. It is particularly satisfactory in the case where the left $R[x, f]$-module $H$ is $x$-torsion-free, for then it turns out that the set of all graded annihilators of submodules of $H$ is in bijective correspondence with a certain set of radical ideals of $R$, and one of the main results of 93 is that this set is finite in the case where $H$ is Artinian as an $R$-module. The theory that emerges has some uncanny similarities to tight closure theory. Use is made of the Hartshorne-Speiser-Lyubeznik Theorem (see R. Hartshorne and R. Speiser [7. Proposition 1.11], G. Lyubeznik [13, Proposition 4.4], and M. Katzman and R. Y. Sharp [12, 1.4 and 1.5]) to pass between a general left $R[x, f]$-module that is Artinian over $R$ and one that is $x$-torsion-free.

In 94 this theory of special annihilator submodules is applied to prove an existence theorem for weak parameter test elements in a Cohen-Macaulay local ring of characteristic $p$. To explain this, I now review some definitions concerning weak test elements.

A $p^{w_{0}}$-weak test element for $R$ (where $w_{0}$ is a non-negative integer) is an element $c^{\prime} \in R^{\circ}$ such that, for every ideal $\mathfrak{b}$ of $R$ and for $r \in R$, it is the case that $r \in \mathfrak{b}^{*}$ if and only if $c^{\prime} r^{p^{n}} \in \mathfrak{b}^{\left[p^{n}\right]}$ for all $n \geq w_{0}$. A $p^{0}$-weak test element is called a test element.

A proper ideal $\mathfrak{a}$ in $R$ is said to be a parameter ideal precisely when it can be generated by ht $\mathfrak{a}$ elements. Parameter ideals play an important rôle in tight closure theory, and Hochster and Huneke introduced the concept of a parameter test element for $R$. A $p^{w_{0}}$-weak parameter test element for $R$ is an element $c^{\prime} \in R^{\circ}$ such that, for every parameter ideal $\mathfrak{b}$ of $R$ and for $r \in R$, it is the case that $r \in \mathfrak{b}^{*}$ if and only if $c^{\prime} r^{p^{n}} \in \mathfrak{b}^{\left[p^{n}\right]}$ for all $n \geq w_{0}$. A $p^{0}$-weak parameter test element is called a parameter test element.

It is a result of Hochster and Huneke [9, Theorem (6.1)(b)] that an algebra of finite type over an excellent local ring of characteristic $p$ has a $p^{w_{0}}$-weak test element for some non-negative integer $w_{0}$; furthermore, such an algebra which is reduced actually has a test element. Of course, a (weak) test element is a (weak) parameter test element.

One of the main results of this paper is Theorem 4.5 which shows that every Cohen-Macaulay local ring of characteristic $p$, even if it is not excellent, has a $p^{w_{0}}$-weak parameter test element for some non-negative integer $w_{0}$.

Lastly, the final $\$ 5$ establishes some connections between the theory developed in this paper and the $F$-stable primes of F. Enescu [3]. 


\section{Graded AnNihilators AND RElated CONCEPtS}

1.1. Notation. Throughout, $R$ will denote a commutative Noetherian ring of prime characteristic $p$. We shall work with the skew polynomial ring $R[x, f]$ associated to $R$ and $f$ in the indeterminate $x$ over $R$. Recall that $R[x, f]$ is, as a left $R$-module, freely generated by $\left(x^{i}\right)_{i \in \mathbb{N}_{0}}$ (I use $\mathbb{N}$ and $\mathbb{N}_{0}$ to denote the set of positive integers and the set of non-negative integers, respectively), and so consists of all polynomials $\sum_{i=0}^{n} r_{i} x^{i}$, where $n \in \mathbb{N}_{0}$ and $r_{0}, \ldots, r_{n} \in R$; however, its multiplication is subject to the rule

$$
x r=f(r) x=r^{p} x \quad \text { for all } r \in R .
$$

Note that $R[x, f]$ can be considered as a positively-graded ring

$$
R[x, f]=\bigoplus_{n=0}^{\infty} R[x, f]_{n},
$$

with $R[x, f]_{n}=R x^{n}$ for all $n \in \mathbb{N}_{0}$. The ring $R[x, f]$ will be referred to as the Frobenius skew polynomial ring over $R$.

Throughout, we shall let $G$ and $H$ denote left $R[x, f]$-modules. The annihilator of $H$ will be denoted by $\operatorname{ann}_{R[x, f]} H$ or $\operatorname{ann}_{R[x, f]}(H)$. Thus

$$
\operatorname{ann}_{R[x, f]}(H)=\{\theta \in R[x, f]: \theta h=0 \text { for all } h \in H\},
$$

and this is a (two-sided) ideal of $R[x, f]$. For a two-sided ideal $\mathfrak{B}$ of $R[x, f]$, we shall use $\operatorname{ann}_{H} \mathfrak{B}$ or $\operatorname{ann}_{H}(\mathfrak{B})$ to denote the annihilator of $\mathfrak{B}$ in $H$. Thus

$$
\operatorname{ann}_{H} \mathfrak{B}=\operatorname{ann}_{H}(\mathfrak{B})=\{h \in H: \theta h=0 \text { for all } \theta \in \mathfrak{B}\},
$$

and this is an $R[x, f]$-submodule of $H$.

1.2. Definition and Remarks. We say that the left $R[x, f]$-module $H$ is $x$-torsionfree if $x h=0$, for $h \in H$, only when $h=0$. The set $\Gamma_{x}(H):=\left\{h \in H: x^{j} h=0\right.$ for some $j \in \mathbb{N}\}$ is an $R[x, f]$-submodule of $H$, called the $x$-torsion submodule of $H$. The $R[x, f]$-module $H / \Gamma_{x}(H)$ is $x$-torsion-free.

1.3. Remark. Let $\mathfrak{B}$ be a subset of $R[x, f]$. It is easy to see that $\mathfrak{B}$ is a graded two-sided ideal of $R[x, f]$ if and only if there is an ascending chain $\left(\mathfrak{b}_{n}\right)_{n \in \mathbb{N}_{0}}$ of ideals of $R$ (which must, of course, eventually be stationary) such that $\mathfrak{B}=\bigoplus_{n \in \mathbb{N}_{0}} \mathfrak{b}_{n} x^{n}$. We shall sometimes denote the ultimate constant value of the ascending sequence $\left(\mathfrak{b}_{n}\right)_{n \in \mathbb{N}_{0}}$ by $\lim _{n \rightarrow \infty} \mathfrak{b}_{n}$.

Note that, in particular, if $\mathfrak{b}$ is an ideal of $R$, then $\mathfrak{b} R[x, f]=\bigoplus_{n \in \mathbb{N}_{0}} \mathfrak{b} x^{n}$ is a graded two-sided ideal of $R[x, f]$. It was noted in Notation [1.1 that the annihilator of a left $R[x, f]$-module is a two-sided ideal.

1.4. Lemma (Y. Yoshino [19, Corollary (2.7)]). The ring $R[x, f]$ satisfies the ascending chain condition on graded two-sided ideals.

Proof. This can be proved by the argument in Yoshino's proof of [19, Corollary $(2.7)]$. 
1.5. Definitions. We define the graded annihilator $\operatorname{gr-ann}_{R[x, f]} H$ of the left $R[x, f]$ module $H$ by

$$
\begin{array}{r}
\operatorname{gr-ann}_{R[x, f]} H=\left\{\sum_{i=0}^{n} r_{i} x^{i} \in R[x, f]: n \in \mathbb{N}_{0}, \text { and } r_{i} \in R, r_{i} x^{i} \in \operatorname{ann}_{R[x, f]} H\right. \\
\text { for all } i=0, \ldots, n\} .
\end{array}
$$

Thus gr-ann ${ }_{R[x, f]} H$ is the largest graded two-sided ideal of $R[x, f]$ contained in

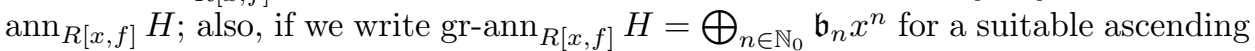
chain $\left(\mathfrak{b}_{n}\right)_{n \in \mathbb{N}_{0}}$ of ideals of $R$, then $\mathfrak{b}_{0}=\left(0:_{R} H\right)$, the annihilator of $H$ as an $R$-module.

We say that an $R[x, f]$-submodule of $H$ is a special annihilator submodule of $H$ if it has the form $\operatorname{ann}_{H}(\mathfrak{B})$ for some graded two-sided ideal $\mathfrak{B}$ of $R[x, f]$. We shall use $\mathcal{A}(H)$ to denote the set of special annihilator submodules of $H$.

1.6. Definition and Remarks. There are some circumstances in which

$$
\operatorname{gr}^{-a n n n_{R[x, f]}} H=\operatorname{ann}_{R[x, f]} H:
$$

for example, this would be the case if $H$ was a $\mathbb{Z}$-graded left $R[x, f]$-module. Work of Y. Yoshino in [19, §2] provides us with further examples.

Following Yoshino [19, Definition (2.1)], we say that $R$ has sufficiently many units precisely when, for each $n \in \mathbb{N}$, there exists $r_{n} \in R$ such that all $n$ elements $\left(r_{n}\right)^{p^{i}}-r_{n}(1 \leq i \leq n)$ are units of $R$. Yoshino proved in [19, Lemma (2.2)] that if either $R$ contains an infinite field, or $R$ is local and has infinite residue field, then $R$ has sufficiently many units. He went on to show in [19, Theorem (2.6)] that, if $R$ has sufficiently many units, then each two-sided ideal of $R[x, f]$ is graded.

Thus if $R$ has sufficiently many units, then gr-ann ${ }_{R[x, f]} H=\operatorname{ann}_{R[x, f]} H$, even if $H$ is not graded.

1.7. Lemma. Let $\mathfrak{B}$ and $\mathfrak{B}^{\prime}$ be graded two-sided ideals of $R[x, f]$ and let $N$ and $N^{\prime}$ be $R[x, f]$-submodules of the left $R[x, f]$-module $H$.

(i) If $\mathfrak{B} \subseteq \mathfrak{B}^{\prime}$, then $\operatorname{ann}_{H}(\mathfrak{B}) \supseteq \operatorname{ann}_{H}\left(\mathfrak{B}^{\prime}\right)$.

(ii) If $N \subseteq N^{\prime}$, then gr-ann $_{R[x, f]} N \supseteq \operatorname{gr-ann}_{R[x, f]} N^{\prime}$.

(iii) We have $\mathfrak{B} \subseteq$ gr-ann $R[x, f]\left(\operatorname{ann}_{H}(\mathfrak{B})\right)$.

(iv) We have $N \subseteq \operatorname{ann}_{H}\left(\operatorname{gr}^{-a n n}{ }_{R[x, f]} N\right)$.

(v) There is an order-reversing bijection, $\Gamma$, from the set $\mathcal{A}(H)$ of special annihilator submodules of $H$ to the set of graded annihilators of submodules of $H$ given by

$$
\Gamma: N \longmapsto \operatorname{gr-ann}_{R[x, f]} N \text {. }
$$

The inverse bijection, $\Gamma^{-1}$, also order-reversing, is given by

$$
\Gamma^{-1}: \mathfrak{B} \longmapsto \operatorname{ann}_{H}(\mathfrak{B}) \text {. }
$$

Proof. Parts (i), (ii), (iii) and (iv) are obvious.

(v) Application of part (i) to the inclusion in part (iii) yields that

$$
\operatorname{ann}_{H}(\mathfrak{B}) \supseteq \operatorname{ann}_{H}\left(\operatorname{gr}-\operatorname{ann}_{R[x, f]}\left(\operatorname{ann}_{H}(\mathfrak{B})\right)\right) ;
$$


however, part (iv) applied to the $R[x, f]$-submodule $\operatorname{ann}_{H}(\mathfrak{B})$ of $H$ yields that

$$
\operatorname{ann}_{H}(\mathfrak{B}) \subseteq \operatorname{ann}_{H}\left(\operatorname{gr}-\operatorname{ann}_{R[x, f]}\left(\operatorname{ann}_{H}(\mathfrak{B})\right)\right) ;
$$

hence $\operatorname{ann}_{H}(\mathfrak{B})=\operatorname{ann}_{H}\left(\operatorname{gr}-\operatorname{ann}_{R[x, f]}\left(\operatorname{ann}_{H}(\mathfrak{B})\right)\right)$. Similar considerations show that

$$
\operatorname{gr}-a n n_{R[x, f]} N=\operatorname{gr-ann}{ }_{R[x, f]}\left(\operatorname{ann}_{H}\left(\operatorname{gr}^{-a n n} n_{R[x, f]} N\right)\right) .
$$

1.8. Remark. It follows from Lemma1.7that, if $N$ is a special annihilator submodule of $H$, then it is the annihilator (in $H$ ) of its own graded annihilator. Likewise, a graded two-sided ideal $\mathfrak{B}$ of $R[x, f]$ which is the graded annihilator of some $R[x, f]$ submodule of $H$ must be the graded annihilator of $\operatorname{ann}_{H}(\mathfrak{B})$.

Much use will be made of the following lemma.

1.9. Lemma. Assume that the left $R[x, f]$-module $G$ is $x$-torsion-free.

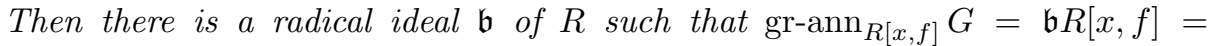
$\bigoplus_{n \in \mathbb{N}_{0}} \mathfrak{b} x^{n}$

Proof. There is a family $\left(\mathfrak{b}_{n}\right)_{n \in \mathbb{N}_{0}}$ of ideals of $R$ such that $\mathfrak{b}_{n} \subseteq \mathfrak{b}_{n+1}$ for all $n \in \mathbb{N}_{0}$

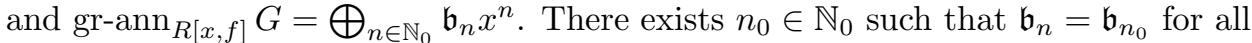
$n \geq n_{0}$. Set $\mathfrak{b}:=\mathfrak{b}_{n_{0}}$. It is enough for us to show that, if $r \in R$ and $e \in \mathbb{N}_{0}$ are such that $r^{p^{e}} \in \mathfrak{b}$, then $r \in \mathfrak{b}_{0}$.

To this end, let $h \in \mathbb{N}$ be such that $h \geq \max \left\{e, n_{0}\right\}$. Then, for all $g \in G$, we have $x^{h} r g=r^{p^{h}} x^{h} g=0$, since $r^{p^{h}} \in \mathfrak{b}=\mathfrak{b}_{h}$. Since $G$ is $x$-torsion-free, it follows that $r G=0$, so that $r \in \mathfrak{b}_{0}$.

1.10. Definition. Assume that the left $R[x, f]$-module $G$ is $x$-torsion-free. An ideal $\mathfrak{b}$ of $R$ is called a $G$-special $R$-ideal if there is an $R[x, f]$-submodule $N$ of $G$ such that gr-ann ${ }_{R[x, f]} N=\mathfrak{b} R[x, f]=\bigoplus_{n \in \mathbb{N}_{0}} \mathfrak{b} x^{n}$. It is worth noting that, then, the ideal $\mathfrak{b}$ is just $\left(0:_{R} N\right)$.

We shall denote the set of $G$-special $R$-ideals by $\mathcal{I}(G)$. Note that, by Lemma 1.9, all the ideals in $\mathcal{I}(G)$ are radical.

We can now combine the results of Lemmas 1.7(v) and 1.9 to obtain the following result, which is fundamental for the work in this paper.

1.11. Proposition. Assume that the left $R[x, f]$-module $G$ is $x$-torsion-free.

There is an order-reversing bijection, $\Delta: \mathcal{A}(G) \longrightarrow \mathcal{I}(G)$, from the set $\mathcal{A}(G)$ of special annihilator submodules of $G$ to the set $\mathcal{I}(G)$ of $G$-special $R$-ideals given by

$$
\Delta: N \longmapsto\left(\operatorname{gr}^{-a_{n}}{ }_{R[x, f]} N\right) \cap R=\left(0:_{R} N\right) .
$$

The inverse bijection, $\Delta^{-1}: \mathcal{I}(G) \longrightarrow \mathcal{A}(G)$, also order-reversing, is given by

$$
\Delta^{-1}: \mathfrak{b} \longmapsto \operatorname{ann}_{G}(\mathfrak{b} R[x, f]) .
$$

When $N \in \mathcal{A}(G)$ and $\mathfrak{b} \in \mathcal{I}(G)$ are such that $\Delta(N)=\mathfrak{b}$, we shall simply say that ' $N$ and $\mathfrak{b}$ correspond'.

1.12. Corollary. Assume that the left $R[x, f]$-module $G$ is $x$-torsion-free.

Then both the sets $\mathcal{A}(G)$ and $\mathcal{I}(G)$ are closed under taking arbitrary intersections. 
Proof. Let $\left(N_{\lambda}\right)_{\lambda \in \Lambda}$ be an arbitrary family of special annihilator submodules of $G$. For each $\lambda \in \Lambda$, let $\mathfrak{b}_{\lambda}$ be the $G$-special $R$-ideal corresponding to $N_{\lambda}$. In view of Proposition 1.11 it is sufficient for us to show that $\bigcap_{\lambda \in \Lambda} N_{\lambda} \in \mathcal{A}(G)$ and $\mathfrak{b}:=\bigcap_{\lambda \in \Lambda} \mathfrak{b}_{\lambda} \in \mathcal{I}(G)$

To prove these, simply note that

$$
\bigcap_{\lambda \in \Lambda} N_{\lambda}=\bigcap_{\lambda \in \Lambda} \operatorname{ann}_{G}\left(\mathfrak{b}_{\lambda} R[x, f]\right)=\operatorname{ann}_{G}\left(\left(\sum_{\lambda \in \Lambda} \mathfrak{b}_{\lambda}\right) R[x, f]\right)
$$

and that $\sum_{\lambda \in \Lambda} N_{\lambda}$ is an $R[x, f]$-submodule of $G$ such that

$$
\operatorname{gr-ann}_{R[x, f]}\left(\sum_{\lambda \in \Lambda} N_{\lambda}\right)=\bigcap_{\lambda \in \Lambda} \operatorname{gr}-a n n_{R[x, f]} N_{\lambda}=\bigcap_{\lambda \in \Lambda}\left(\mathfrak{b}_{\lambda} R[x, f]\right)=\mathfrak{b} R[x, f] .
$$

1.13. Remark. Suppose that the left $R[x, f]$-module $G$ is $x$-torsion-free.

It is worth pointing out now that, since $R$ is Noetherian, so that the set $\mathcal{I}(G)$ of $G$-special $R$-ideals satisfies the ascending chain condition, it is a consequence of Proposition 1.11 that the set $\mathcal{A}(G)$ of special annihilator submodules of $G$, partially ordered by inclusion, satisfies the descending chain condition. This is the case even if $G$ is not finitely generated. Note that (by [19, Theorem (1.3)]) the (noncommutative) ring $R[x, f]$ is neither left nor right Noetherian if $\operatorname{dim} R>0$.

\section{EXAmples RELEVANT to THE THEORY OF Tight ClOSURE}

The purpose of this section is to present some motivating examples, from the theory of tight closure, of some of the concepts introduced in \$1. Throughout this section, we shall again employ the notation of Notation 1.1, and $\mathfrak{a}$ will always denote an ideal of $R$. Recall that the Frobenius closure $\mathfrak{a}^{F}$ of $\mathfrak{a}$ is the ideal of $R$ defined by

$$
\mathfrak{a}^{F}:=\left\{r \in R: \text { there exists } n \in \mathbb{N}_{0} \text { such that } r^{p^{n}} \in \mathfrak{a}^{\left[p^{n}\right]}\right\} .
$$

2.1. Remark. Let $\left(\mathfrak{b}_{n}\right)_{n \in \mathbb{N}_{0}}$ be a family of ideals of $R$ such that $\mathfrak{b}_{n} \subseteq f^{-1}\left(\mathfrak{b}_{n+1}\right)$ for all $n \in \mathbb{N}_{0}$. Then $\bigoplus_{n \in \mathbb{N}_{0}} \mathfrak{b}_{n} x^{n}$ is a graded left ideal of $R[x, f]$, and so we may form the graded left $R[x, f]$-module $R[x, f] / \bigoplus_{n \in \mathbb{N}_{0}} \mathfrak{b}_{n} x^{n}$. This may be viewed as $\bigoplus_{n \in \mathbb{N}_{0}} R / \mathfrak{b}_{n}$, where, for $r \in R$ and $n \in \mathbb{N}_{0}$, the result of multiplying the element $r+\mathfrak{b}_{n}$ of the $n$-th component by $x$ is the element $r^{p}+\mathfrak{b}_{n+1}$ of the $(n+1)$-th component.

Note that the left $R[x, f]$-module $R[x, f] / \bigoplus_{n \in \mathbb{N}_{0}} \mathfrak{b}_{n} x^{n}$ is $x$-torsion-free if and only if $\mathfrak{b}_{n}=f^{-1}\left(\mathfrak{b}_{n+1}\right)$ for all $n \in \mathbb{N}_{0}$, that is, if and only if $\left(\mathfrak{b}_{n}\right)_{n \in \mathbb{N}_{0}}$ is an $f$ sequence in the sense of [16, Definition 4.1(ii)].

2.2. Notation. Since $R[x, f] \mathfrak{a}=\bigoplus_{n \in \mathbb{N}_{0}} \mathfrak{a}^{\left[p^{n}\right]} x^{n}$, we can view the graded left $R[x, f]$ module

$$
R[x, f] / R[x, f] \mathfrak{a}
$$

as $\bigoplus_{n \in \mathbb{N}_{0}} R / \mathfrak{a}^{\left[p^{n}\right]}$ in the manner described in Remark 2.1] We shall denote the graded left $R[x, f]$-module $\bigoplus_{n \in \mathbb{N}_{0}} R / \mathfrak{a}^{\left[p^{n}\right]}$ by $H(\mathfrak{a})$.

Recall from [16, 4.1(iii)] that $\left(\left(\mathfrak{a}^{\left[p^{n}\right]}\right)^{F}\right)_{n \in \mathbb{N}_{0}}$ is the canonical $f$-sequence associated to $\mathfrak{a}$. We shall denote $\bigoplus_{n \in \mathbb{N}_{0}} R /\left(\mathfrak{a}^{\left[p^{n}\right]}\right)^{F}$, considered as a graded left $R[x, f]$ module in the manner described in Remark 2.1 by $G(\mathfrak{a})$. Note that $G(\mathfrak{a})$ is $x$ torsion-free. 
2.3. Lemma. With Notation 2.2 , we have $\Gamma_{x}(H(\mathfrak{a}))=\bigoplus_{n \in \mathbb{N}_{0}}\left(\mathfrak{a}^{\left[p^{n}\right]}\right)^{F} / \mathfrak{a}^{\left[p^{n}\right]}$, so that there is an isomorphism of graded left $R[x, f]$-modules

$$
H(\mathfrak{a}) / \Gamma_{x}(H(\mathfrak{a})) \cong G(\mathfrak{a})
$$

Proof. Let $n \in \mathbb{N}_{0}$ and $r \in R$. Then the element $r+\mathfrak{a}^{\left[p^{n}\right]}$ of the $n$-th component of $H(\mathfrak{a})$ belongs to $\Gamma_{x}(H(\mathfrak{a}))$ if and only if there exists $m \in \mathbb{N}_{0}$ such that $x^{m}\left(r+\mathfrak{a}^{\left[p^{n}\right]}\right)=$ $r^{p^{m}}+\left(\mathfrak{a}^{\left[p^{n}\right]}\right)^{\left[p^{m}\right]}=0$, that is, if and only if $r \in\left(\mathfrak{a}^{\left[p^{n}\right]}\right)^{F}$.

2.4. Proposition. We use Notation 2.2.

Suppose that there exists a $p^{w_{0}}$-weak test element $c$ for $R$, for some $w_{0} \in \mathbb{N}_{0}$. Then

(i) $\operatorname{ann}_{H(\mathfrak{a})}\left(\bigoplus_{n \geq w_{0}} R c x^{n}\right)=\bigoplus_{n \in \mathbb{N}_{0}}\left(\mathfrak{a}^{\left[p^{n}\right]}\right)^{*} / \mathfrak{a}^{\left[p^{n}\right]}$;

(ii) $\operatorname{ann}_{G(\mathfrak{a})}\left(\bigoplus_{n \in \mathbb{N}_{0}} R c x^{n}\right)=\bigoplus_{n \in \mathbb{N}_{0}}\left(\mathfrak{a}^{\left[p^{n}\right]}\right)^{*} /\left(\mathfrak{a}^{\left[p^{n}\right]}\right)^{F}$.

Proof. (i) Let $j \in \mathbb{N}_{0}$ and $r \in R$. Then the element $r+\mathfrak{a}^{\left[p^{j}\right]}$ of the $j$-th component of $H(\mathfrak{a})$ belongs to $\operatorname{ann}_{H(\mathfrak{a})}\left(\bigoplus_{n \geq w_{0}} R c x^{n}\right)$ if and only if $c r^{p^{n}} \in\left(\mathfrak{a}^{\left[p^{j}\right]}\right)^{\left[p^{n}\right]}$ for all $n \geq w_{0}$, that is, if and only if $r \in\left(\mathfrak{a}^{\left[p^{j}\right]}\right)^{*}$.

(ii) By part (i),

$$
\bigoplus_{n \in \mathbb{N}_{0}}\left(\mathfrak{a}^{\left[p^{n}\right]}\right)^{*} /\left(\mathfrak{a}^{\left[p^{n}\right]}\right)^{F} \subseteq \operatorname{ann}_{G(\mathfrak{a})}\left(\bigoplus_{n \geq w_{0}} R c x^{n}\right) .
$$

Note that $\operatorname{ann}_{G(\mathfrak{a})}\left(\bigoplus_{n \geq w_{0}} R c x^{n}\right)$ is a graded $R[x, f]$-submodule of $G(\mathfrak{a})$. Let $j \in \mathbb{N}_{0}$ and $r \in R$ be such that $r+\left(\mathfrak{a}^{\left[p^{j}\right]}\right)^{F}$ belongs to the $j$-th component of $\operatorname{ann}_{G(\mathfrak{a})}\left(\bigoplus_{n \geq w_{0}} R c x^{n}\right)$. Then, for all $n \geq w_{0}$, we have $c r p^{p^{n}} \in\left(\mathfrak{a}^{\left[p^{j+n}\right]}\right)^{F}=$ $\left(\left(\mathfrak{a}^{\left[p^{j}\right]}\right)^{\left[p^{n}\right]}\right)^{F}$. Therefore, by [12, Lemma 0.1], we have $r \in\left(\mathfrak{a}^{\left[p^{j}\right]}\right)^{*}$.

It follows from this that

$$
\bigoplus_{n \in \mathbb{N}_{0}}\left(\mathfrak{a}^{\left[p^{n}\right]}\right)^{*} /\left(\mathfrak{a}^{\left[p^{n}\right]}\right)^{F}=\operatorname{ann}_{G(\mathfrak{a})}\left(\bigoplus_{n \geq w_{0}} R c x^{n}\right),
$$

and so $\bigoplus_{n \in \mathbb{N}_{0}}\left(\mathfrak{a}^{\left[p^{n}\right]}\right)^{*} /\left(\mathfrak{a}^{\left[p^{n}\right]}\right)^{F}$ is a special annihilator submodule of the $x$-torsionfree graded left $R[x, f]$-module $G(\mathfrak{a})$. Let $\mathfrak{b}$ be the $G(\mathfrak{a})$-special $R$-ideal corresponding to this member of $\mathcal{A}(G(\mathfrak{a}))$. The above-displayed equation shows that $R c \subseteq \mathfrak{b}$. Hence, by Proposition 1.11,

$$
\begin{aligned}
\bigoplus_{n \in \mathbb{N}_{0}}\left(\mathfrak{a}^{\left[p^{n}\right]}\right)^{*} /\left(\mathfrak{a}^{\left[p^{n}\right]}\right)^{F} & =\operatorname{ann}_{G(\mathfrak{a})}\left(\bigoplus_{n \geq w_{0}} R c x^{n}\right) \supseteq \operatorname{ann}_{G(\mathfrak{a})}\left(\bigoplus_{n \in \mathbb{N}_{0}} R c x^{n}\right) \\
& \supseteq \operatorname{ann}_{G(\mathfrak{a})}\left(\bigoplus_{n \in \mathbb{N}_{0}} \mathfrak{b} x^{n}\right)=\bigoplus_{n \in \mathbb{N}_{0}}\left(\mathfrak{a}^{\left[p^{n}\right]}\right)^{*} /\left(\mathfrak{a}^{\left[p^{n}\right]}\right)^{F}
\end{aligned}
$$

2.5. Definition. The weak test ideal $\tau^{\prime}(R)$ of $R$ is defined to be the ideal generated by 0 and all weak test elements for $R$. (By a 'weak test element' for $R$ we mean a $p^{w_{0}}$-weak test element for $R$ for some $w_{0} \in \mathbb{N}_{0}$.)

It is easy to see that each element of $\tau^{\prime}(R) \cap R^{\circ}$ is a weak test element for $R$.

2.6. Theorem. We use Notation 2.2. Suppose that there exists a $p^{w_{0}}$-weak test element $c$ for $R$, for some $w_{0} \in \mathbb{N}_{0}$.

Let $H$ be the positively-graded left $R[x, f]$-module given by

$$
H:=\bigoplus_{\mathfrak{a} \text { is an ideal of } R} H(\mathfrak{a})=\bigoplus_{\mathfrak{a} \text { is an ideal of } R}\left(\bigoplus_{n \in \mathbb{N}_{0}} R / \mathfrak{a}^{\left[p^{n}\right]}\right) .
$$




$$
\text { Set } T:=\bigoplus_{\mathfrak{a} \text { is an ideal of } R}\left(\bigoplus_{n \in \mathbb{N}_{0}}\left(\mathfrak{a}^{\left[p^{n}\right]}\right)^{*} / \mathfrak{a}^{\left[p^{n}\right]}\right) .
$$

(i) Then $T=\operatorname{ann}_{H}\left(\bigoplus_{n \geq w_{0}} R c x^{n}\right)$, and so is a special annihilator submodule of $H$.

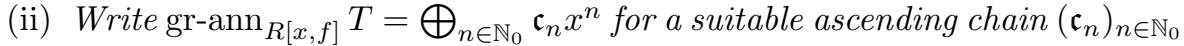
of ideals of $R$. Then $\lim _{n \rightarrow \infty} \mathfrak{c}_{n}=\tau^{\prime}(R)$, the weak test ideal for $R$.

(iii) Furthermore, $T$ contains every special annihilator submodule $T^{\prime}$ of $H$ for

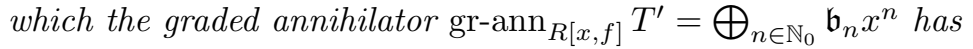

$$
\operatorname{ht}\left(\lim _{n \rightarrow \infty} \mathfrak{b}_{n}\right) \geq 1 \text {. }
$$

(The height of the improper ideal $R$ is considered to be $\infty$.)

Proof. (i) This is immediate from Proposition 2.4(i).

(ii) Write $\mathfrak{c}:=\lim _{n \rightarrow \infty} \mathfrak{c}_{n}$. Since there exists a weak test element for $R$, the ideal $\tau^{\prime}(R)$ can be generated by finitely many weak test elements for $R$, say by $c_{i}(i=1, \ldots, h)$, where $c_{i}$ is a $p^{w_{i}}$-weak test element for $R$ (for $\left.i=1, \ldots, h\right)$. Set $\widetilde{w}=\max \left\{w_{1}, \ldots, w_{h}\right\}$. It is immediate from part (i) that $\bigoplus_{n \geq \widetilde{w}} \tau^{\prime}(R) x^{n} \subseteq$ $\operatorname{gr}-\operatorname{ann}_{R[x, f]} T$, and so $\tau^{\prime}(R) \subseteq \mathfrak{c}$. Therefore ht $\mathfrak{c} \geq 1$, so that $\mathfrak{c} \cap R^{\circ} \neq \emptyset$ by prime avoidance, and $\mathfrak{c}$ can be generated by its elements in $R^{\circ}$.

There exists $m_{0} \in \mathbb{N}_{0}$ such that $\mathfrak{c}_{n}=\mathfrak{c}$ for all $n \geq m_{0}$. Let $c^{\prime} \in \mathfrak{c} \cap R^{\circ}$. Thus $T$ is annihilated by $c^{\prime} x^{n}$ for all $n \geq m_{0}$; therefore, for each ideal $\mathfrak{a}$ of $R$, and for all $r \in \mathfrak{a}^{*}$, we have $c^{\prime} r^{p^{n}} \in \mathfrak{a}^{\left[p^{n}\right]}$ for all $n \geq m_{0}$, so that $c^{\prime}$ is a $p^{m_{0}}$-weak test element for $R$. Therefore $c^{\prime} \in \tau^{\prime}(R)$. Since $\mathfrak{c}$ can be generated by elements in $\mathfrak{c} \cap R^{\circ}$, it follows that $\mathfrak{c} \subseteq \tau^{\prime}(R)$.

(iii) Since $T^{\prime}=\operatorname{ann}_{H}\left(\bigoplus_{n \in \mathbb{N}_{0}} \mathfrak{b}_{n} x^{n}\right)$, it follows that

$$
T^{\prime}=\bigoplus_{\mathfrak{a} \text { is an ideal of } R}\left(\bigoplus_{n \in \mathbb{N}_{0}} \mathfrak{a}_{n} / \mathfrak{a}^{\left[p^{n}\right]}\right),
$$

where, for each ideal $\mathfrak{a}$ of $R$ and each $n \in \mathbb{N}_{0}$, the ideal $\mathfrak{a}_{n}$ of $R$ contains $\mathfrak{a}^{\left[p^{n}\right]}$. Suppose that $\lim _{n \rightarrow \infty} \mathfrak{b}_{n}=\mathfrak{b}$ and that $v_{0} \in \mathbb{N}_{0}$ is such that $\mathfrak{b}_{n}=\mathfrak{b}$ for all $n \geq v_{0}$. Since ht $\mathfrak{b} \geq 1$, there exists $\bar{c} \in \mathfrak{b} \cap R^{\circ}$, by prime avoidance. Let $\mathfrak{a}$ be an ideal of $R$ and let $n \in \mathbb{N}_{0}$. Then, for each $r \in \mathfrak{a}_{n}$, the element $r+\mathfrak{a}^{\left[p^{n}\right]}$ of the $n$-th component of $H(\mathfrak{a})$ is annihilated by $\bar{c} x^{j}$ for all $j \geq v_{0}$. This means that $\bar{c} r^{p^{j}} \in\left(\mathfrak{a}^{\left[p^{n}\right]}\right)^{\left[p^{j}\right]}$ for all $j \geq v_{0}$, so that $r \in\left(\mathfrak{a}^{\left[p^{n}\right]}\right)^{*}$. Therefore $T^{\prime} \subseteq T$.

2.7. Theorem. We use Notation 2.2, Suppose that there exists a $p^{w_{0}}$-weak test element $c$ for $R$, for some $w_{0} \in \mathbb{N}_{0}$.

Let $G$ be the positively-graded $x$-torsion-free left $R[x, f]$-module given by

$$
G:=\bigoplus_{\mathfrak{a} \text { is an ideal of } R} G(\mathfrak{a})=\bigoplus_{\mathfrak{a} \text { is an ideal of } R}\left(\bigoplus_{n \in \mathbb{N}_{0}} R /\left(\mathfrak{a}^{\left[p^{n}\right]}\right)^{F}\right) .
$$

$$
\text { Set } U:=\bigoplus_{\mathfrak{a} \text { is an ideal of } R}\left(\bigoplus_{n \in \mathbb{N}_{0}}\left(\mathfrak{a}^{\left[p^{n}\right]}\right)^{*} /\left(\mathfrak{a}^{\left[p^{n}\right]}\right)^{F}\right) \text {. }
$$

(i) Then $U=\operatorname{ann}_{G}\left(\bigoplus_{n \in \mathbb{N}_{0}} R c x^{n}\right)$, and so is a special annihilator submodule of $G$.

(ii) Let $\mathfrak{b}$ be the $G$-special $R$-ideal corresponding to $U$. Then $\mathfrak{b}$ is the smallest member of $\mathcal{I}(G)$ of positive height. 
Proof. (i) This is immediate from Proposition 2.4(ii).

(ii) Note that $R c \subseteq \mathfrak{b}$, by part (i); therefore ht $\mathfrak{b} \geq 1$. To complete the proof, we show that, if $\mathfrak{b}^{\prime} \in \mathcal{I}(G)$ has ht $\mathfrak{b}^{\prime} \geq 1$, then $\mathfrak{b} \subseteq \mathfrak{b}^{\prime}$. By prime avoidance, there exists $\widetilde{c} \in \mathfrak{b}^{\prime} \cap R^{\circ}$. Let $U^{\prime} \in \mathcal{A}(G)$ correspond to $\mathfrak{b}^{\prime}$ (in the correspondence of Proposition 1.11). Since $U^{\prime}=\operatorname{ann}_{G} \mathfrak{b}^{\prime} R[x, f]$, it follows that

$$
U^{\prime}=\bigoplus_{\mathfrak{a} \text { is an ideal of } R}\left(\bigoplus_{n \in \mathbb{N}_{0}} \mathfrak{a}_{n} /\left(\mathfrak{a}^{\left[p^{n}\right]}\right)^{F}\right),
$$

where, for each ideal $\mathfrak{a}$ of $R$ and each $n \in \mathbb{N}_{0}$, the ideal $\mathfrak{a}_{n}$ of $R$ contains $\left(\mathfrak{a}^{\left[p^{n}\right]}\right)^{F}$. Let $\mathfrak{a}$ be an ideal of $R$ and let $n \in \mathbb{N}_{0}$. Then, for each $r \in \mathfrak{a}_{n}$, the element $r+\left(\mathfrak{a}^{\left[p^{n}\right]}\right)^{F}$ of the $n$-th component of $G(\mathfrak{a})$ is annihilated by $\widetilde{c} x^{j}$ for all $j \geq 0$. This means that $\widetilde{c} r^{p^{j}} \in\left(\left(\mathfrak{a}^{\left[p^{n}\right]}\right)^{\left[p^{j}\right]}\right)^{F}$ for all $j \geq 0$, so that $r \in\left(\mathfrak{a}^{\left[p^{n}\right]}\right)^{*}$ by [12, Lemma 0.1(i)]. Therefore $U^{\prime} \subseteq U$, so that $\mathfrak{b}^{\prime} \supseteq \mathfrak{b}$.

\section{Properties of SPeCial anNihilator Submodules IN THE $x$-TORSION-FREE CASE}

Throughout this section, we shall employ Notation 1.1. The aim is to develop the theory of special annihilator submodules of an $x$-torsion-free left $R[x, f]$-module.

3.1. Lemma. Suppose that $G$ is $x$-torsion-free. Let $N$ be a special annihilator submodule of $G$. Then the left $R[x, f]$-module $G / N$ is also $x$-torsion-free.

Proof. By Lemma 1.9 and Proposition 1.11, there is a radical ideal $\mathfrak{b}$ of $R$ such that $N=\operatorname{ann}_{G}(\mathfrak{b} R[x, f])$. Let $g \in G$ be such that $x g \in N$. Therefore, for all $r \in \mathfrak{b}$ and all $j \in \mathbb{N}_{0}$, we have $r x^{j}(x g)=0$, that is, $r x^{j+1} g=0$. Also, for $r \in \mathfrak{b}$, since $r(x g)=0$, we have $x(r g)=r^{p} x g=0$, and so $r g=0$ because $G$ is $x$-torsion-free. Thus $g \in \operatorname{ann}_{G}\left(\bigoplus_{n \in \mathbb{N}_{0}} \mathfrak{b} x^{n}\right)=N$. It follows that $G / N$ is $x$-torsion-free.

3.2. Lemma. Suppose that $G$ is $x$-torsion-free. Let $\mathfrak{a}$ be an ideal of $R$, and set $L:=\operatorname{ann}_{G}(\mathfrak{a} R[x, f]) \in \mathcal{A}(G)$. Then $L=\operatorname{ann}_{G}(\sqrt{\mathfrak{a}} R[x, f])$.

Proof. Let $\mathfrak{d} \in \mathcal{I}(G)$ correspond to $L$. Note that $\mathfrak{d}$ is radical, by Lemma 1.9] also, $\mathfrak{a} \subseteq \mathfrak{d}$. Hence $\mathfrak{a} \subseteq \sqrt{\mathfrak{a}} \subseteq \sqrt{\mathfrak{d}}=\mathfrak{d}$. Since $\operatorname{ann}_{G}(\mathfrak{a} R[x, f])=\operatorname{ann}_{G}(\mathfrak{d} R[x, f])$, we must have $\operatorname{ann}_{G}(\mathfrak{a} R[x, f])=\operatorname{ann}_{G}(\sqrt{\mathfrak{a}} R[x, f])$.

3.3. Proposition. Suppose that $G$ is $x$-torsion-free. Let $\mathfrak{a}$ be an ideal of $R$, and set

$$
L:=\operatorname{ann}_{G}(\mathfrak{a} R[x, f]) \in \mathcal{A}(G) .
$$

Note that $G / L$ is $x$-torsion-free, by Lemma 3.1. Let $N$ be an $R$-submodule of $G$ such that $L \subseteq N \subseteq G$.

(i) If $N=\operatorname{ann}_{G}(\mathfrak{b} R[x, f]) \in \mathcal{A}(G)$, where $\mathfrak{b}$ is an ideal of $R$ contained in $\mathfrak{a}$, then

$$
N / L=\operatorname{ann}_{G / L}((\mathfrak{b}: \mathfrak{a}) R[x, f]) \in \mathcal{A}(G / L) .
$$

Furthermore, if the ideal in $\mathcal{I}(G)$ corresponding to $N$ is $\mathfrak{b}$, then $(\mathfrak{b}: \mathfrak{a})$ is the ideal in $\mathcal{I}(G / L)$ corresponding to $N / L$.

(ii) If $N / L=\operatorname{ann}_{G / L}(\mathfrak{c} R[x, f]) \in \mathcal{A}(G / L)$, where $\mathfrak{c}$ is an ideal of $R$, then

$$
N=\operatorname{ann}_{G}(\mathfrak{a} c R[x, f])=\operatorname{ann}_{G}((\mathfrak{a} \cap \mathfrak{c}) R[x, f]) \in \mathcal{A}(G) .
$$

Furthermore, if $\mathfrak{a}$ is the ideal in $\mathcal{I}(G)$ corresponding to $L$ and $\mathfrak{c}$ is the ideal in $\mathcal{I}(G / L)$ corresponding to $N / L$, then $\mathfrak{a} \cap \mathfrak{c}$ is the ideal in $\mathcal{I}(G)$ corresponding to $N$. 
(iii) There is an order-preserving bijection from $\{N \in \mathcal{A}(G): N \supseteq L\}$ to $\mathcal{A}(G / L)$ given by $N \mapsto N / L$.

Proof. By Lemma 3.1, the left $R[x, f]$-module $G / L$ is $x$-torsion-free.

(i) Let $g \in N$. Let $i, j \in \mathbb{N}_{0}$ and $r \in(\mathfrak{b}: \mathfrak{a}), u \in \mathfrak{a}$. Then $u x^{i}\left(r x^{j} g\right)=$ $u r^{p^{i}} x^{i+j} g=0$ because $u r^{p^{i}} \in \mathfrak{b}$ and $\mathfrak{b} x^{i+j}$ annihilates $N$. This is true for all $i \in \mathbb{N}_{0}$ and $u \in \mathfrak{a}$. Therefore $r x^{j} g \in \operatorname{ann}_{G}(\mathfrak{a} R[x, f])=L$. Since this is true for all $j \in \mathbb{N}_{0}$ and $r \in(\mathfrak{b}: \mathfrak{a})$, we see that $N / L \subseteq \operatorname{ann}_{G / L}((\mathfrak{b}: \mathfrak{a}) R[x, f])$.

Now suppose that $g \in G$ is such that $g+L \in \operatorname{ann}_{G / L}((\mathfrak{b}: \mathfrak{a}) R[x, f])$. Let $r \in \mathfrak{b}$ and $i \in \mathbb{N}_{0}$. Then $r \in(\mathfrak{b}: \mathfrak{a})$ and so $r x^{i+1}$ annihilates $g+L \in G / L$. Hence $r x^{i+1} g \in L$. Since $\mathfrak{b} \subseteq \mathfrak{a}$, we see that $r^{p-1} r x^{i+1} g=0$, so that $x r x^{i} g=0$. As $G$ is $x$-torsion-free, it follows that $r x^{i} g=0$. As this is true for all $r \in \mathfrak{b}$ and $i \in \mathbb{N}_{0}$, we see that $g \in \operatorname{ann}_{G}(\mathfrak{b} R[x, f])=N$. Hence $N / L=\operatorname{ann}_{G / L}((\mathfrak{b}: \mathfrak{a}) R[x, f])$.

To prove the final claim, we have to show that

$$
\operatorname{gr}^{-\operatorname{ann}_{R[x, f]}}(N / L)=\bigoplus_{n \in \mathbb{N}_{0}}(\mathfrak{b}: \mathfrak{a}) x^{n},
$$

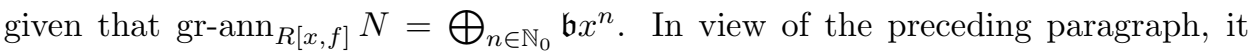
remains only to show that

$$
\operatorname{gr}^{-\operatorname{ann}_{R[x, f]}(N / L) \subseteq(\mathfrak{b}: \mathfrak{a}) R[x, f] .}
$$

Let $r \in R$ be such that $r x^{i} \in \operatorname{gr}^{-a n n_{R[x, f]}}(N / L)$ for all $i \in \mathbb{N}_{0}$. Let $g \in N$. Then $r x^{i} g \in L$ for all $i \in \mathbb{N}_{0}$, and so ar $x^{i} g=0$ for all $i \in \mathbb{N}_{0}$. As this is true for all $g \in N$ and for all $i \in \mathbb{N}_{0}$, it follows that $\mathfrak{a} r \subseteq\left(\operatorname{gr}^{-a n n}{ }_{R[x, f]} N\right) \cap R=\mathfrak{b}$. Hence $r \in(\mathfrak{b}: \mathfrak{a})$.

(ii) Let $g \in N$. Then $u x^{i} g \in L$ for all $u \in \mathfrak{c}$ and $i \in \mathbb{N}_{0}$, and so $r u x^{i} g=0$ for all $r \in \mathfrak{a}, u \in \mathfrak{c}$ and $i \in \mathbb{N}_{0}$. Hence $N \subseteq \operatorname{ann}_{G}\left(\bigoplus_{n \in \mathbb{N}_{0}} \mathfrak{a c} x^{n}\right)=\operatorname{ann}_{G}(\mathfrak{a c} R[x, f])$.

Now let $g \in \operatorname{ann}_{G}(\mathfrak{a} \mathfrak{c} R[x, f])$. Then, for all $r \in \mathfrak{a}, u \in \mathfrak{c}$ and $i, j \in \mathbb{N}_{0}$, we have $r x^{i}\left(u x^{j} g\right)=r u^{p^{i}} x^{i+j} g=0$, and so $u x^{j} g \in L$ for all $u \in \mathfrak{c}$ and $j \in \mathbb{N}_{0}$. Hence $g+L \in$ $\operatorname{ann}_{G / L}\left(\bigoplus_{n \in \mathbb{N}_{0}} \mathfrak{c} x^{n}\right)=N / L$, and $g \in N$. It follows that $N=\operatorname{ann}_{G}(\mathfrak{a} \mathfrak{c} R[x, f])$. Also, by Lemma 3.2, we have

$$
\operatorname{ann}_{G}(\mathfrak{a c} R[x, f])=\operatorname{ann}_{G}((\mathfrak{a} \cap \mathfrak{c}) R[x, f]),
$$

because $\mathfrak{a}$ and $\mathfrak{a} \cap \mathfrak{c}$ have the same radical.

To prove the final claim, we have to show that $\operatorname{gr}^{-a n n}{ }_{R[x, f]} N=(\mathfrak{a} \cap \mathfrak{c}) R[x, f]$, given that

$$
\operatorname{gr}^{-\operatorname{ann}_{R[x, f]}}(N / L)=\mathfrak{c} R[x, f] \quad \text { and } \quad \operatorname{gr} \operatorname{ann}_{R[x, f]}(L)=\mathfrak{a} R[x, f] .
$$

In view of the preceding paragraph, it remains only to show that gr-ann ${ }_{R[x, f]} N \subseteq$ $(\mathfrak{a} \cap \mathfrak{c}) R[x, f]$. However, this is clear, because

$$
\operatorname{gr-ann}_{R[x, f]} N \subseteq \operatorname{gr-ann}_{R[x, f]} L \cap \operatorname{gr}_{-a n n}^{R[x, f]}(N / L) .
$$

(iii) This is now immediate from parts (i) and (ii).

3.4. Remark. It follows from Proposition 3.3(ii) (and with the hypotheses and notation thereof) that, if $\mathfrak{a}$ is an ideal of $R$ and $L:=\operatorname{ann}_{G}(\mathfrak{a} R[x, f])$, then

$$
\operatorname{ann}_{G / L}(\mathfrak{a} R[x, f])=0 .
$$

Because the special $R$-ideals introduced in Definition 1.10 are radical, the following lemma will be very useful. 
3.5. Lemma. Let $\mathfrak{a}$ and $\mathfrak{b}$ be proper radical ideals of $R$, and let their (unique) minimal primary decompositions be

$$
\mathfrak{a}=\mathfrak{r}_{1} \cap \ldots \cap \mathfrak{r}_{k} \cap \mathfrak{p}_{1} \cap \ldots \cap \mathfrak{p}_{t} \cap \mathfrak{p}_{1}^{\prime} \cap \ldots \cap \mathfrak{p}_{u}^{\prime}
$$

and

$$
\mathfrak{b}=\mathfrak{r}_{1} \cap \ldots \cap \mathfrak{r}_{k} \cap \mathfrak{q}_{1} \cap \ldots \cap \mathfrak{q}_{v} \cap \mathfrak{q}_{1}^{\prime} \cap \ldots \cap \mathfrak{q}_{w}^{\prime},
$$

where the notation is such that

$$
\left\{\mathfrak{p}_{1}, \ldots, \mathfrak{p}_{t}, \mathfrak{p}_{1}^{\prime}, \ldots, \mathfrak{p}_{u}^{\prime}\right\} \cap\left\{\mathfrak{q}_{1}, \ldots, \mathfrak{q}_{v}, \mathfrak{q}_{1}^{\prime}, \ldots, \mathfrak{q}_{w}^{\prime}\right\}=\emptyset,
$$

and such that none of $\mathfrak{p}_{1}, \ldots, \mathfrak{p}_{t}$ contains an associated prime of $\mathfrak{b}$, each of $\mathfrak{p}_{1}^{\prime}, \ldots, \mathfrak{p}_{u}^{\prime}$ contains an associated prime of $\mathfrak{b}$, none of $\mathfrak{q}_{1}, \ldots, \mathfrak{q}_{v}$ contains an associated prime of $\mathfrak{a}$, and each of $\mathfrak{q}_{1}^{\prime}, \ldots, \mathfrak{q}_{w}^{\prime}$ contains an associated prime of $\mathfrak{a}$. (Note that some, but not all, of the integers $k, t$ and $u$ might be zero; a similar comment applies to the primary decomposition of $\mathfrak{b}$.) Then

(i) $\mathfrak{a} \cap \mathfrak{b}=\mathfrak{r}_{1} \cap \ldots \cap \mathfrak{r}_{k} \cap \mathfrak{p}_{1} \cap \ldots \cap \mathfrak{p}_{t} \cap \mathfrak{q}_{1} \cap \ldots \cap \mathfrak{q}_{v}$ is the minimal primary decomposition;

(ii) if $\mathfrak{a} \not \subset \mathfrak{b}$, the equation $(\mathfrak{b}: \mathfrak{a})=\mathfrak{q}_{1} \cap \ldots \cap \mathfrak{q}_{v}$ gives the minimal primary decomposition.

Proof. (i) Each of $\mathfrak{p}_{1}^{\prime}, \ldots, \mathfrak{p}_{u}^{\prime}$ must contain one of $\mathfrak{q}_{1}, \ldots, \mathfrak{q}_{v}$; likewise, each of $\mathfrak{q}_{1}^{\prime}, \ldots$, $\mathfrak{q}_{w}^{\prime}$ must contain one of $\mathfrak{p}_{1}, \ldots, \mathfrak{p}_{t}$. The claim then follows easily.

(ii) Since $(\mathfrak{b}: \mathfrak{a})=(\mathfrak{b} \cap \mathfrak{a}: \mathfrak{a})$, it is clear from part (i) that

$$
\mathfrak{q}_{1} \cap \ldots \cap \mathfrak{q}_{v} \subseteq(\mathfrak{b}: \mathfrak{a}) .
$$

Now let $r \in(\mathfrak{b}: \mathfrak{a})=(\mathfrak{b} \cap \mathfrak{a}: \mathfrak{a})$. Then, for each $i=1, \ldots, v$, we have $r \mathfrak{a} \subseteq \mathfrak{q}_{i}$, whereas $\mathfrak{a} \not \mathfrak{q} \mathfrak{q}_{i}$; hence $r \in \mathfrak{q}_{i}$ because $\mathfrak{q}_{i}$ is prime.

3.6. Theorem. Suppose that $G$ is $x$-torsion-free. Let $N:=\operatorname{ann}_{G}(\mathfrak{b} R[x, f]) \in$ $\mathcal{A}(G)$, where the ideal $\mathfrak{b} \in \mathcal{I}(G)$ corresponds to $N$. Assume that $N \neq 0$, and let $\mathfrak{b}=\mathfrak{p}_{1} \cap \ldots \cap \mathfrak{p}_{t}$ be the minimal primary decomposition of the (radical) ideal $\mathfrak{b}$.

Suppose that $t>1$, and consider any partition $\{1, \ldots, t\}=U \cup V$, where $U$ and $V$ are two non-empty disjoint sets. Set $\mathfrak{a}=\bigcap_{i \in U} \mathfrak{p}_{i}$ and $\mathfrak{c}=\bigcap_{i \in V} \mathfrak{p}_{i}$. Let $L:=\operatorname{ann}_{G}(\mathfrak{a} R[x, f]) \in \mathcal{A}(G)$. Then

(i) $0 \subset L \subset N$ (the symbol ' $\subset$ ' is reserved to denote strict inclusion);

(ii) $N / L=\operatorname{ann}_{G / L}(\mathfrak{c} R[x, f]) \in \mathcal{A}(G / L)$ with corresponding ideal $\mathfrak{c} \in \mathcal{I}(G / L)$; and

(iii) gr-ann $\operatorname{gnc}_{R[x, f]} L=\mathfrak{a} R[x, f]$, so that $\mathfrak{a} \in \mathcal{I}(G)$ corresponds to $L$.

Proof. (i) It is clear that $L \subseteq N$. Suppose that $L=0$ and seek a contradiction. Let $g \in N$. Let $i, j \in \mathbb{N}_{0}$ and $r \in \mathfrak{c}, u \in \mathfrak{a}$. Then $u x^{i}\left(r x^{j} g\right)=u r^{p^{i}} x^{i+j} g=0$ because $u r^{p^{i}} \in \mathfrak{b}$ and $\mathfrak{b} x^{i+j}$ annihilates $N$. This is true for all $i \in \mathbb{N}_{0}$ and $u \in \mathfrak{a}$. Therefore $r x^{j} g \in \operatorname{ann}_{G}\left(\bigoplus_{n \in \mathbb{N}_{0}} \mathfrak{a} x^{n}\right)=L=0$. It follows that $\bigoplus_{n \in \mathbb{N}_{0}} \mathfrak{c} x^{n} \subseteq \operatorname{gr}^{-a n n}{ }_{R[x, f]} N=$ $\bigoplus_{n \in \mathbb{N}_{0}} \mathfrak{b} x^{n}$, so that $\mathfrak{c} \subseteq \mathfrak{b}$. But $\mathfrak{b} \subseteq \mathfrak{c}$, and so $\mathfrak{c}=\mathfrak{b}$. However, this contradicts the fact that $\mathfrak{b}=\mathfrak{p}_{1} \cap \ldots \cap \mathfrak{p}_{t}$ is the unique minimal primary decomposition of $\mathfrak{b}$. Therefore $L \neq 0$.

Now suppose that $L=N$ and again seek a contradiction. Then $\bigoplus_{n \in \mathbb{N}_{0}} \mathfrak{a} x^{n} \subseteq$ $\operatorname{gr}^{-a n n}{ }_{R[x, f]} N=\bigoplus_{n \in \mathbb{N}_{0}} \mathfrak{b} x^{n}$, so that $\mathfrak{a} \subseteq \mathfrak{b}$. But $\mathfrak{b} \subseteq \mathfrak{a}$, and so $\mathfrak{a}=\mathfrak{b}$, and this again leads to a contradiction. Therefore $L \neq N$.

(ii) Since $\mathfrak{b} \subseteq \mathfrak{a}$, it is immediate from Proposition 3.3(i) that

$$
N / L=\operatorname{ann}_{G / L}((\mathfrak{b}: \mathfrak{a}) R[x, f]) \in \mathcal{A}(G / L)
$$


and that the ideal $(\mathfrak{b}: \mathfrak{a}) \in \mathcal{I}(G / L)$ corresponds to $N / L$. However, it follows from Lemma 3.5(ii) that $(\mathfrak{b}: \mathfrak{a})=\bigcap_{i \in V} \mathfrak{p}_{i}=\mathfrak{c}$.

(iii) Let $\mathfrak{d} \in \mathcal{I}(G)$ correspond to $L$. Note that $\mathfrak{a}=\bigcap_{i \in U} \mathfrak{p}_{i} \subseteq \mathfrak{d}$. By Proposition 3.3(i), the ideal in $\mathcal{I}(G / L)$ corresponding to $N / L$ is $(\mathfrak{b}: \mathfrak{d})$. Therefore, by part (ii), we have $(\mathfrak{b}: \mathfrak{d})=\mathfrak{c}$. But, by Proposition 3.3(ii), the ideal in $\mathcal{I}(G)$ corresponding to $N$ is $\mathfrak{d} \cap \mathfrak{c}$. Therefore $\mathfrak{b}=\mathfrak{d} \cap \mathfrak{c}$, and so $\mathfrak{d} \neq R$.

Now $\mathfrak{d}$ is a radical ideal of $R$. By Lemma 3.5(i), each $\mathfrak{p}_{j}$, for $j \in U$, is an associated prime of $\mathfrak{d}$. Hence $\mathfrak{d} \subseteq \bigcap_{j \in U} \mathfrak{p}_{j}=\mathfrak{a}$. But we already know that $\mathfrak{a} \subseteq \mathfrak{d}$, and so $\mathfrak{d}=\mathfrak{a}$.

3.7. Corollary. Suppose that $G$ is $x$-torsion-free. Then the set of $G$-special $R$-ideals is precisely the set of all finite intersections of prime $G$-special $R$-ideals (provided one includes the empty intersection, $R$, which corresponds to the zero special annihilator submodule of $G$ ). In symbols,

$$
\mathcal{I}(G)=\left\{\mathfrak{p}_{1} \cap \ldots \cap \mathfrak{p}_{t}: t \in \mathbb{N}_{0} \text { and } \mathfrak{p}_{1}, \ldots, \mathfrak{p}_{t} \in \mathcal{I}(G) \cap \operatorname{Spec}(R)\right\} .
$$

Proof. By Corollary 1.12, the set $\mathcal{I}(G)$ is closed under taking intersections. A proper ideal $\mathfrak{a} \in \mathcal{I}(G)$ is radical, and it follows from Theorem 3.6 that each (necessarily prime) primary component of $\mathfrak{a}$ also belongs to $\mathcal{I}(G)$. This is enough to complete the proof.

3.8. Lemma. Suppose that $G$ is $x$-torsion-free. Let $\mathfrak{p}$ be a maximal member of $\mathcal{I}(G) \backslash\{R\}$ with respect to inclusion, and let $L \in \mathcal{A}(G)$ be the corresponding special annihilator submodule of $G$. Thus $L$ is a minimal member of the set of non-zero special annihilator submodules of $G$.

Then $\mathfrak{p}$ is prime, and any non-zero $g \in L$ satisfies $\operatorname{gr}^{-a n n_{R[x, f]}} R[x, f] g=$ $\mathfrak{p} R[x, f]$.

Proof. It follows from Corollary 3.7 that $\mathfrak{p}$ is prime.

Since $R[x, f] g$ is a non-zero $R[x, f]$-submodule of $L$, there is a proper radical ideal $\mathfrak{a} \in \mathcal{I}(G)$ such that

$$
\mathfrak{a} R[x, f]=\operatorname{gr}^{-a n n n_{R[x, f]}} R[x, f] g \supseteq \operatorname{gr}_{-a n n_{R[x, f]}} L=\mathfrak{p} R[f, x] .
$$

Since $\mathfrak{p}$ is a maximal member of $\mathcal{I}(G) \backslash\{R\}$, we must have $\mathfrak{a}=\mathfrak{p}$.

Our next major aim is to show that, in the situation of Corollary 3.7, the set $\mathcal{I}(G)$ is finite if $G$ has the property that, for each special annihilator submodule $L$ of $G$ (including $0=\operatorname{ann}_{G} R[x, f]$ ), the $x$-torsion-free residue class module $G / L$ (see Lemma 3.1) does not contain, as an $R[x, f]$-submodule, an infinite direct sum of non-zero special annihilator submodules of $G / L$. This may seem rather a complicated hypothesis, and so we point out now that it is satisfied if $G$ is a Noetherian or Artinian left $R[x, f]$-module, and therefore if $G$ is a Noetherian or Artinian $R$ module. These ideas will be applied, later in the paper, to an example in which $G$ is Artinian as an $R$-module.

The following lemma will be helpful in an inductive argument in the proof of Theorem 3.10 .

3.9. Lemma. Suppose that $G$ is $x$-torsion-free, and that the set $\mathcal{I}(G) \backslash\{R\}$ is nonempty and has finitely many maximal members: suppose that there are $n$ of these and denote them by $\mathfrak{p}_{1}, \ldots, \mathfrak{p}_{n}$. (The ideals $\mathfrak{p}_{1}, \ldots, \mathfrak{p}_{n}$ are prime, by Lemma 3.8.) 
Let $L:=\operatorname{ann}_{G}\left(\mathfrak{p}_{1} \cap \cdots \cap \mathfrak{p}_{n}\right) R[x, f]$. Then the left $R[x, f]$-module $G / L$ is $x$ torsion-free, and

$$
\mathcal{I}(G / L) \cap \operatorname{Spec}(R)=\mathcal{I}(G) \cap \operatorname{Spec}(R) \backslash\left\{\mathfrak{p}_{1}, \ldots, \mathfrak{p}_{n}\right\} .
$$

Proof. Note that $\bigcap_{i=1}^{n} \mathfrak{p}_{i} \in \mathcal{I}(G)$, by Corollary 1.12, Therefore gr-ann $\cos _{R, f]} L=$ $\left(\bigcap_{i=1}^{n} \mathfrak{p}_{i}\right) R[x, f]$ and $L$ corresponds to $\bigcap_{i=1}^{n} \mathfrak{p}_{i}$.

That $G / L$ is $x$-torsion-free follows from Lemma 3.1. By Proposition 3.3(iii),

$$
\mathcal{A}(G / L)=\{N / L: N \in \mathcal{A}(G) \text { and } L \subseteq N\} .
$$

Let $N \in \mathcal{A}(G)$ with $L \subset N$, and let $\mathfrak{b} \in \mathcal{I}(G)$ correspond to $N$. Note that $\mathfrak{b} \subset$ $\bigcap_{i=1}^{n} \mathfrak{p}_{i}$, and that no associated prime of $\mathfrak{b}$ can properly contain any of $\mathfrak{p}_{1}, \ldots, \mathfrak{p}_{n}$. Therefore the minimal primary decomposition of the radical ideal $\mathfrak{b}$ will have the form

$$
\mathfrak{b}=\left(\bigcap_{i \in I} \mathfrak{p}_{i}\right) \cap \mathfrak{q}_{1} \cap \ldots \cap \mathfrak{q}_{v},
$$

where $I$ is some (possibly empty) subset of $\{1, \ldots, n\}$ and none of $\mathfrak{q}_{1}, \ldots, \mathfrak{q}_{v}$ contains any of $\mathfrak{p}_{1}, \ldots, \mathfrak{p}_{n}$. Note that $\mathfrak{q}_{1}, \ldots, \mathfrak{q} v$ must all belong to

$$
\mathcal{I}(G) \cap \operatorname{Spec}(R) \backslash\left\{\mathfrak{p}_{1}, \ldots, \mathfrak{p}_{n}\right\} .
$$

Proposition 3.3(i), this time used in conjunction with Lemma 3.5)(ii), now shows that $N / L \in \mathcal{A}(G / L)$, and the ideal of $\mathcal{I}(G / L)$ corresponding to $N / L$ is

$$
\left(\mathfrak{b}: \mathfrak{p}_{1} \cap \cdots \cap \mathfrak{p}_{n}\right)=\mathfrak{q}_{1} \cap \ldots \cap \mathfrak{q}_{v} .
$$

Note also that, if $\mathfrak{q} \in \mathcal{I}(G) \cap \operatorname{Spec}(R) \backslash\left\{\mathfrak{p}_{1}, \ldots, \mathfrak{p}_{n}\right\}$ and

$$
J:=\left\{j \in\{1, \ldots, n\}: \mathfrak{p}_{j} \not \supset \mathfrak{q}\right\},
$$

then $\mathfrak{c}:=\left(\bigcap_{j \in J} \mathfrak{p}_{j}\right) \cap \mathfrak{q} \in \mathcal{I}(G)$ and $\mathfrak{c} \subset \bigcap_{i=1}^{n} \mathfrak{p}_{i}$. It now follows from Corollary 3.7 that

$$
\mathcal{I}(G / L) \cap \operatorname{Spec}(R)=\mathcal{I}(G) \cap \operatorname{Spec}(R) \backslash\left\{\mathfrak{p}_{1}, \ldots, \mathfrak{p}_{n}\right\},
$$

as required.

3.10. Theorem. Suppose that $G$ is $x$-torsion-free. Assume that $G$ has the property that, for each special annihilator submodule $L$ of $G$ (including $0=\operatorname{ann}_{G} R[x, f]$ ), the $x$-torsion-free residue class module $G / L$ does not contain, as an $R[x, f]$-submodule, an infinite direct sum of non-zero special annihilator submodules of $G / L$.

Then the set $\mathcal{I}(G)$ of $G$-special $R$-ideals is finite.

Proof. By Corollary 3.7, it is enough for us to show that the set $\mathcal{I}(G) \cap \operatorname{Spec}(R)$ is finite; we may suppose that the latter set is not empty, so that it has maximal members with respect to inclusion. In the first part of the proof, we show that $\mathcal{I}(G) \cap \operatorname{Spec}(R)$ has only finitely many such maximal members.

Let $\left(\mathfrak{p}_{\lambda}\right)_{\lambda \in \Lambda}$ be a labelling of the set of maximal members of $\mathcal{I}(G) \cap \operatorname{Spec}(R)$, arranged so that $\mathfrak{p}_{\lambda} \neq \mathfrak{p}_{\mu}$ whenever $\lambda$ and $\mu$ are different elements of $\Lambda$. For each $\lambda \in \Lambda$, let $S_{\lambda}$ be the member of $\mathcal{A}(G)$ corresponding to $\mathfrak{p}_{\lambda}$.

Consider $\lambda, \mu \in \Lambda$ with $\lambda \neq \mu$. By Lemma 3.8, a non-zero $g \in S_{\lambda} \cap S_{\mu}$ would have to satisfy gr-ann $\operatorname{ang}_{R[x]} R[x, f] g=\mathfrak{p}_{\lambda} R[x, f]=\mathfrak{p}_{\mu} R[x, f]$. Since $\mathfrak{p}_{\lambda} \neq \mathfrak{p}_{\mu}$, this is impossible. Therefore $S_{\lambda} \cap S_{\mu}=0$ and the sum $S_{\lambda}+S_{\mu}$ is direct. 
Suppose, inductively, that $n \in \mathbb{N}$ and we have shown that, whenever $\lambda_{1}, \ldots, \lambda_{n}$ are $n$ distinct members of $\Lambda$, then the sum $\sum_{i=1}^{n} S_{\lambda_{i}}$ is direct. We can now use Lemma 3.8 to see that, if $g_{i} \in S_{\lambda_{i}}$ for $i=1, \ldots, n$, then

$$
\operatorname{gr}-a n n_{R[x, f]} R[x, f]\left(g_{1}+\cdots+g_{n}\right)=\bigcap_{\substack{i=1 \\ g_{i} \neq 0}}^{n} \mathfrak{p}_{\lambda_{i}} R[x, f],
$$

and then to deduce that, for $\lambda_{n+1} \in \Lambda \backslash\left\{\lambda_{1}, \ldots, \lambda_{n}\right\}$, we must have

$$
\left(\bigoplus_{i=1}^{n} S_{\lambda_{i}}\right) \bigcap S_{\lambda_{n+1}}=0
$$

so that the sum $S_{\lambda_{1}}+\cdots+S_{\lambda_{n}}+S_{\lambda_{n+1}}$ is direct.

It follows that the sum $\sum_{\lambda \in \Lambda} S_{\lambda}$ is direct; since each $S_{\lambda}$ is non-zero, the hypothesis about $G / 0$ (that is, about $G$ ) ensures that $\Lambda$ is finite.

We have thus shown that $\mathcal{I}(G) \cap \operatorname{Spec}(R)$ has only finitely many maximal members. Note that $\max \{$ ht $\mathfrak{p}: \mathfrak{p}$ is a maximal member of $\mathcal{I}(G) \cap \operatorname{Spec}(R)\}$ is an upper bound for the lengths of chains

$$
\mathfrak{p}_{0} \subset \mathfrak{p}_{1} \subset \cdots \subset \mathfrak{p}_{w}
$$

of prime ideals in $\mathcal{I}(G) \cap \operatorname{Spec}(R)$. We argue by induction on the maximum $t$ of these lengths. When $t=0$, all members of $\mathcal{I}(G) \cap \operatorname{Spec}(R)$ are maximal members of that set, and so, by the first part of this proof, $\mathcal{I}(G) \cap \operatorname{Spec}(R)$ is finite. Now suppose that $t>0$, and that it has been proved that $\mathcal{I}(G) \cap \operatorname{Spec}(R)$ is finite for smaller values of $t$.

We know that there are only finitely many maximal members of $\mathcal{I}(G) \cap \operatorname{Spec}(R)$; suppose that there are $n$ of these and denote them by $\mathfrak{p}_{1}, \ldots, \mathfrak{p}_{n}$. Let $L:=$ $\operatorname{ann}_{G}\left(\mathfrak{p}_{1} \cap \cdots \cap \mathfrak{p}_{n}\right) R[x, f]$. We can now use Lemma 3.9 to deduce that the left $R[x, f]$-module $G / L$ is $x$-torsion-free and

$$
\mathcal{I}(G / L) \cap \operatorname{Spec}(R)=\mathcal{I}(G) \cap \operatorname{Spec}(R) \backslash\left\{\mathfrak{p}_{1}, \ldots, \mathfrak{p}_{n}\right\} .
$$

It follows from this and Proposition 3.3(ii) that the inductive hypothesis can be applied to $G / L$, and so we can deduce that the set

$$
\mathcal{I}(G) \cap \operatorname{Spec}(R) \backslash\left\{\mathfrak{p}_{1}, \ldots, \mathfrak{p}_{n}\right\}
$$

is finite. Hence $\mathcal{I}(G) \cap \operatorname{Spec}(R)$ is a finite set and the inductive step is complete.

3.11. Corollary. Suppose that the left $R[x, f]$-module $G$ is $x$-torsion-free and either Artinian or Noetherian as an R-module. Then the set $\mathcal{I}(G)$ of $G$-special R-ideals is finite.

3.12. Theorem. Suppose that $G$ is $x$-torsion-free and that the set $\mathcal{I}(G)$ of $G$-special $R$-ideals is finite. Then there exists a (uniquely determined) ideal $\mathfrak{b} \in \mathcal{I}(G)$ with the properties that ht $\mathfrak{b} \geq 1$ (the improper ideal $R$ is considered to have infinite height) and $\mathfrak{b} \subset \mathfrak{c}$ for every other ideal $\mathfrak{c} \in \mathcal{I}(G)$ with ht $\mathfrak{c} \geq 1$. Furthermore, for $g \in G$, the following statements are equivalent:

(i) $g$ is annihilated by $\mathfrak{b} R[x, f]=\bigoplus_{n \in \mathbb{N}_{0}} \mathfrak{b} x^{n}$;

(ii) there exists $c \in R^{\circ} \cap \mathfrak{b}$ such that $c x^{n} g=0$ for all $n \gg 0$;

(iii) there exists $c \in R^{\circ}$ such that $c x^{n} g=0$ for all $n \gg 0$. 
Proof. By Corollary 3.7, we have

$$
\mathcal{I}(G)=\left\{\mathfrak{p}_{1} \cap \ldots \cap \mathfrak{p}_{t}: t \in \mathbb{N}_{0} \text { and } \mathfrak{p}_{1}, \ldots, \mathfrak{p}_{t} \in \mathcal{I}(G) \cap \operatorname{Spec}(R)\right\} .
$$

Since $\mathcal{I}(G)$ is finite, it is immediate that

$$
\mathfrak{b}:=\bigcap_{\substack{\mathfrak{p} \in \mathcal{I}(G) \cap \operatorname{Spec}(R) \\ \text { ht } \mathfrak{p} \geq 1}} \mathfrak{p}
$$

is the smallest ideal in $\mathcal{I}(G)$ of height greater than 0 . Since $h t \mathfrak{b} \geq 1$, so that there exists $c \in \mathfrak{b} \cap R^{\circ}$ by prime avoidance, it is clear that (i) $\Rightarrow$ (ii) and (ii) $\Rightarrow$ (iii).

(iii) $\Rightarrow$ (i) Let $n_{0} \in \mathbb{N}_{0}$ and $c \in R^{\circ}$ be such that $c x^{n} g=0$ for all $n \geq n_{0}$. Then, for all $j \in \mathbb{N}_{0}$, we have $x^{n_{0}} c x^{j} g=c^{p^{n_{0}}} x^{n_{0}+j} g=0$, so that $c x^{j} g=0$ because $G$ is $x$-torsion-free.

Therefore $g \in \operatorname{ann}_{G}(R c R[x, f])$. Now $\operatorname{ann}_{G}(R c R[x, f]) \in \mathcal{A}(G)$ : let $\mathfrak{a} \in \mathcal{I}(G)$ be the corresponding $G$-special $R$-ideal. Since $c \in \mathfrak{a}$, we must have ht $\mathfrak{a} \geq 1$. Therefore $\mathfrak{b} \subseteq \mathfrak{a}$, by definition of $\mathfrak{b}$, and so

$$
g \in \operatorname{ann}_{G}(R c R[x, f])=\operatorname{ann}_{G}(\mathfrak{a} R[x, f]) \subseteq \operatorname{ann}_{G}(\mathfrak{b} R[x, f]) .
$$

Corollary 3.11 and Theorem 3.12 give hints about how this work will be exploited, in Section 4 below, to obtain results in the theory of tight closure. The aim is to apply Corollary 3.11 and Theorem 3.12 to $H_{\mathfrak{m}}^{d}(R) / \Gamma_{x}\left(H_{\mathfrak{m}}^{d}(R)\right)$, where $(R, \mathfrak{m})$ is a local ring of dimension $d>0$; the local cohomology module $H_{\mathfrak{m}}^{d}(R)$, which is well known to be Artinian as an $R$-module, carries a natural structure as a left $R[x, f]$-module. The passage between $H_{\mathfrak{m}}^{d}(R)$ and its $x$-torsion-free residue class $R[x, f]$-module $H_{\mathfrak{m}}^{d}(R) / \Gamma_{x}\left(H_{\mathfrak{m}}^{d}(R)\right)$ is facilitated by the following extension, due to G. Lyubeznik, of a result of R. Hartshorne and R. Speiser. It shows that, when $R$ is local, an $x$-torsion left $R[x, f]$-module which is Artinian (that is, 'cofinite' in the terminology of Hartshorne and Speiser) as an $R$-module exhibits a certain uniformity of behaviour.

3.13. Theorem (G. Lyubeznik [13, Proposition 4.4] and compare Hartshorne and Speiser [7, Proposition 1.11]). Suppose that $(R, \mathfrak{m})$ is local, and let $H$ be a left $R[x, f]$-module which is Artinian as an $R$-module. Then there exists $e \in \mathbb{N}_{0}$ such that $x^{e} \Gamma_{x}(H)=0$.

Hartshorne and Speiser first proved this result in the case where $R$ is local and contains its residue field which is perfect. Lyubeznik applied his theory of $F$ modules to obtain the result without restriction on the local ring $R$ of characteristic $p$.

3.14. Definition. Suppose that $(R, \mathfrak{m})$ is local, and let $H$ be a left $R[x, f]$-module which is Artinian as an $R$-module. By the Hartshorne-Speiser-Lyubeznik Theorem 3.13, there exists $e \in \mathbb{N}_{0}$ such that $x^{e} \Gamma_{x}(H)=0$ : we call the smallest such $e$ the Hartshorne-Speiser-Lyubeznik number, or HSL-number for short, of $H$.

It will be helpful to have available an extension of this idea.

3.15. Definition. We say that the left $R[x, f]$-module $H$ admits an $H S L$-number if there exists $e \in \mathbb{N}_{0}$ such that $x^{e} \Gamma_{x}(H)=0$; then we call the smallest such $e$ the HSL-number of $H$. 
We have seen above in Theorem 3.13 and Definition 3.14 that if $H$ is Artinian as an $R$-module, then it admits an HSL-number. Note also that if $H$ is Noetherian as an $R$-module, then it admits an HSL-number, because $\Gamma_{x}(H)$ is an $R[x, f]$ submodule of $H$, and so is an $R$-submodule and therefore finitely generated.

3.16. Corollary. Suppose that the left $R[x, f]$-module $H$ admits an HSL-number $m_{0}$, and that the $x$-torsion-free left $R[x, f]$-module $G:=H / \Gamma_{x}(H)$ has only finitely many $G$-special $R$-ideals. Let $\mathfrak{b}$ be the smallest ideal in $\mathcal{I}(G)$ of positive height (see Theorem 3.12). For $h \in H$, the following statements are equivalent:

(i) $h$ is annihilated by $\bigoplus_{n \geq m_{0}} \mathfrak{b}^{\left[p^{m_{0}}\right]} x^{n}$;

(ii) there exists $c \in R^{\circ} \cap \mathfrak{b}$ such that $c x^{n} h=0$ for all $n \geq m_{0}$;

(iii) there exists $c \in R^{\circ} \cap \mathfrak{b}$ such that $c x^{n} h=0$ for all $n \gg 0$;

(iv) there exists $c \in R^{\circ}$ such that $c x^{n} h=0$ for all $n \gg 0$.

Proof. Since $\mathfrak{b} \cap R^{\circ} \neq 0$ by prime avoidance, it is clear that (i) $\Rightarrow$ (ii), (ii) $\Rightarrow$ (iii) and (iii) $\Rightarrow$ (iv).

(iv) $\Rightarrow$ (i) Since $c x^{n}\left(h+\Gamma_{x}(H)\right)=0$ in $G$ for all $n \gg 0$, it follows from Theorem 3.12 that $h+\Gamma_{x}(H)$ is annihilated by $\mathfrak{b} R[x, f]$. Therefore, for all $r \in \mathfrak{b}$ and $j \in \mathbb{N}_{0}$, we have $r x^{j}\left(h+\Gamma_{x}(H)\right)=0$, so that $r x^{j} h \in \Gamma_{x}(H)$ and $r^{p^{m_{0}}} x^{m_{0}+j} h=x^{m_{0}} r x^{j} h=$ 0. Therefore $h \in \operatorname{ann}_{H}\left(\bigoplus_{n \geq m_{0}} \mathfrak{b}^{\left[p^{m_{0}}\right]} x^{n}\right)$.

\section{Applications to tight Closure}

The aim of this section is to apply results from Section 3 to the theory of tight closure in the local ring $(R, \mathfrak{m})$ of dimension $d>0$. As was mentioned in Section 3. we shall be concerned with the top local cohomology module $H_{\mathfrak{m}}^{d}(R)$, which has a natural structure as a left $R[x, f]$-module, and its $x$-torsion-free residue class module $H_{\mathfrak{m}}^{d}(R) / \Gamma_{x}\left(H_{\mathfrak{m}}^{d}(R)\right)$. The (well-known) left $R[x, f]$-module structure carried by $H_{\mathfrak{m}}^{d}(R)$ is described in detail in [12, 2.1 and 2.3].

4.1. Reminder. Suppose that $(R, \mathfrak{m})$ is a local ring of dimension $d>0$. The abovementioned natural left $R[x, f]$-module structure carried by $H_{\mathfrak{m}}^{d}(R)$ is independent of any choice of a system of parameters for $R$. However, if one does choose a system of parameters $a_{1}, \ldots, a_{d}$ for $R$, then one can obtain a quite concrete representation of the local cohomology module $H_{\mathrm{m}}^{d}(R)$ and, through this, an explicit formula for the effect of multiplication by the indeterminate $x \in R[x, f]$ on an element of $H_{\mathfrak{m}}^{d}(R)$.

Denote by $a_{1}, \ldots, a_{d}$ a system of parameters for $R$.

(i) Represent $H_{\mathfrak{m}}^{d}(R)$ as the $d$-th cohomology module of the Cech complex of $R$ with respect to $a_{1}, \ldots, a_{d}$, that is, as the residue class module of $R_{a_{1} \ldots a_{d}}$ modulo the image, under the Čch complex 'differentiation' map, of $\bigoplus_{i=1}^{d} R_{a_{1} \ldots a_{i-1} a_{i+1} \ldots a_{d}}$. See [1, $\left.\S 5.1\right]$. We use '[ ]' to denote natural images of elements of $R_{a_{1} \ldots a_{d}}$ in this residue class module. Note that, for $i \in\{1, \ldots, d\}$, we have

$$
\left[\frac{a_{i}^{k}}{\left(a_{1} \ldots a_{d}\right)^{k}}\right]=0 \quad \text { for all } k \in \mathbb{N}_{0} .
$$

Denote the product $a_{1} \ldots a_{d}$ by $a$. A typical element of $H_{\mathfrak{m}}^{d}(R)$ can be represented as $\left[r / a^{j}\right]$ for some $r \in R$ and $j \in \mathbb{N}_{0}$; moreover, for $r, r_{1} \in R$ and $j, j_{1} \in \mathbb{N}_{0}$, we have $\left[r / a^{j}\right]=\left[r_{1} / a^{j_{1}}\right]$ if and only if there exists $k \in \mathbb{N}_{0}$ 
such that $k \geq \max \left\{j, j_{1}\right\}$ and $a^{k-j} r-a^{k-j_{1}} r_{1} \in\left(a_{1}^{k}, \ldots, a_{d}^{k}\right) R$. In particular, if $a_{1}, \ldots, a_{d}$ form an $R$-sequence (that is, if $R$ is Cohen-Macaulay), then $\left[r / a^{j}\right]=0$ if and only if $r \in\left(a_{1}^{j}, \ldots, a_{d}^{j}\right) R$, by [15, Theorem 3.2], for example.

(ii) The left $R[x, f]$-module structure on $H_{\mathfrak{m}}^{d}(R)$ is such that

$$
x\left[\frac{r}{\left(a_{1} \ldots a_{d}\right)^{j}}\right]=\left[\frac{r^{p}}{\left(a_{1} \ldots a_{d}\right)^{j p}}\right] \quad \text { for all } r \in R \text { and } j \in \mathbb{N}_{0} .
$$

The reader might like to consult [12, 2.3] for more details, and should in any case note that this left $R[x, f]$-module structure does not depend on the choice of system of parameters $a_{1}, \ldots, a_{d}$.

4.2. Remark. Let the situation and notation be as in Reminder 4.1. Here we relate the left $R[x, f]$-module structure on $H:=H_{\mathfrak{m}}^{d}(R)$ described in Reminder 4.1 to the tight closure in $H$ of its zero submodule. See [ 8 , Definition (8.2)] for the definition of the tight closure in an $R$-module of one of its submodules. Let $n \in \mathbb{N}_{0}$.

(i) The $n$-th component $R x^{n}$ of $R[x, f]$ is isomorphic, as an $(R, R)$-bimodule, to $R$ considered as a left $R$-module in the natural way and as a right $R$-module via $f^{n}$, the $n$-th power of the Frobenius ring homomorphism. Let $L$ be a submodule of the $R$-module $M$. It follows that an element $m \in M$ belongs to $L_{M}^{*}$, the tight closure of $L$ in $M$, if and only if there exists $c \in R^{\circ}$ such that $c x^{n} \otimes m$ belongs, for all $n \gg 0$, to the image of $R[x, f] \otimes_{R} L$ in $R[x, f] \otimes_{R} M$ under the map induced by inclusion.

(ii) Let $S$ be a multiplicatively closed subset of $R$. It is straightforward to check that there is an isomorphism of $R$-modules

$$
\gamma_{n}: R x^{n} \otimes_{R} S^{-1} R \stackrel{\cong}{\longrightarrow} S^{-1} R
$$

for which $\gamma_{n}\left(b x^{n} \otimes(r / s)\right)=b r^{p^{n}} / s^{p^{n}}$ for all $b, r \in R$ and $s \in S$; the inverse of $\gamma_{n}$ satisfies $\left(\gamma_{n}\right)^{-1}(r / s)=r s^{p^{n}-1} x^{n} \otimes(1 / s)$ for all $r \in R$ and $s \in S$.

(iii) Now represent $H:=H_{\mathfrak{m}}^{d}(R)$ as the $d$-th cohomology module of the C̆ech complex of $R$ with respect to the system of parameters $a_{1}, \ldots, a_{d}$, as in Reminder 4.1(i). We can use isomorphisms like those described in part (ii), together with the right exactness of tensor product, to see that (when we think of $H$ simply as an $R$-module) there is an isomorphism of $R$-modules $\delta_{n}: R x^{n} \otimes_{R} H \stackrel{\cong}{\longrightarrow} H$ for which

$\delta_{n}\left(b x^{n} \otimes\left[\frac{r}{\left(a_{1} \ldots a_{d}\right)^{j}}\right]\right)=\left[\frac{b r^{p^{n}}}{\left(a_{1} \ldots a_{d}\right)^{j p^{n}}}\right] \quad$ for all $b, r \in R$ and $j \in \mathbb{N}_{0}$.

Thus, in terms of the natural left $R[x, f]$-module structure on $H$, we have $\delta_{n}\left(b x^{n} \otimes h\right)=b x^{n} h$ for all $b \in R$ and $h \in H$.

(iv) It thus follows that, for $h \in H$, we have $h \in 0_{H}^{*}$ if and only if there exists $c \in R^{\circ}$ such that $c x^{n} h=0$ for all $n \gg 0$.

(v) Observe that $\Gamma_{x}(H) \subseteq 0_{H}^{*}$.

(vi) Suppose that $(R, \mathfrak{m})$ is Cohen-Macaulay, and use the notation of part (iii) again; write $a:=a_{1} \ldots a_{d}$. Let $r \in R, j \in \mathbb{N}_{0}$, and let $h:=\left[r / a^{j}\right]$ in $H$. It follows from Reminder $4.1(\mathrm{i})$ that $r \in\left(\left(a_{1}^{j}, \ldots, a_{d}^{j}\right) R\right)^{*}$ if and only if there exists $c \in R^{\circ}$ such that $c x^{n} h=0$ for all $n \gg 0$. Thus, by part (iv) above, $r \in\left(\left(a_{1}^{j}, \ldots, a_{d}^{j}\right) R\right)^{*}$ if and only if $h \in 0_{H}^{*}$. 
(vii) Let the situation and notation be as in part (vi) above. Then the $R$ homomorphism $\nu_{j}: R /\left(a_{1}^{j}, \ldots, a_{d}^{j}\right) R \longrightarrow H$ for which $\nu_{j}\left(r^{\prime}+\left(a_{1}^{j}, \ldots, a_{d}^{j}\right) R\right)$ $=\left[r^{\prime} / a^{j}\right]$ for all $r^{\prime} \in R$ is a monomorphism (by Reminder 4.1(i)). Furthermore, the induced homogeneous $R[x, f]$-homomorphism

$$
R[x, f] \otimes_{R} \nu_{j}: R[x, f] \otimes_{R}\left(R /\left(a_{1}^{j}, \ldots, a_{d}^{j}\right) R\right) \longrightarrow R[x, f] \otimes_{R} H
$$

of graded left $R[x, f]$-modules is also a monomorphism: this is because a homogeneous element of $\operatorname{Ker}\left(R[x, f] \otimes_{R} \nu_{j}\right)$ must have the form

$$
r^{\prime} x^{k} \otimes\left(1+\left(a_{1}^{j}, \ldots, a_{d}^{j}\right) R\right)
$$

for some $r^{\prime} \in R$ and $k \in \mathbb{N}_{0}$; since $r^{\prime} x^{k} \otimes\left[1 / a^{j}\right]=0$, it follows from Remark 4.2(iii) and Reminder 4.1(i) that $r^{\prime} \in\left(a_{1}^{j p^{k}}, \ldots, a_{d}^{j p^{k}}\right) R$, so that $r^{\prime} x^{k} \otimes\left(1+\left(a_{1}^{j}, \ldots, a_{d}^{j}\right) R\right)=0$.

4.3. Lemma. Suppose that $(R, \mathfrak{m})$ is a local ring of dimension $d>0$; set $H:=$ $H_{\mathfrak{m}}^{d}(R)$ and $G:=H / \Gamma_{x}(H)$. Let $h \in H$. Then the following statements are equivalent:

(i) $h \in 0_{H}^{*}$;

(ii) $h+\Gamma_{x}(H) \in 0_{G}^{*}$;

(iii) there exists $c \in R^{\circ}$ such that $c x^{n}\left(h+\Gamma_{x}(H)\right)=0$ in $G$ for all $n \gg 0$;

(iv) there exists $c \in R^{\circ}$ such that $c x^{n}\left(h+\Gamma_{x}(H)\right)=0$ in $G$ for all $n \geq 0$.

Proof. Let $m_{0}$ denote the HSL-number of $H$ (see Definition 3.14).

(i) $\Rightarrow$ (ii) This is immediate from the fact that $0_{H}^{*} \subseteq\left(\Gamma_{x}(H)\right)_{H}^{*}$ once it is recalled from [8, Remark (8.4)] that $h+\Gamma_{x}(H) \in 0_{G}^{*}$ if and only if $h \in\left(\Gamma_{x}(H)\right)_{H}^{*}$.

(ii) $\Rightarrow$ (iii) Suppose that $h+\Gamma_{x}(H) \in 0_{G}^{*}$, so that $h \in\left(\Gamma_{x}(H)\right)_{H}^{*}$. Under the isomorphism $\delta_{n}: R x^{n} \otimes_{R} H \stackrel{\cong}{\cong} H$ of Remark 4.2(iii) (where $n \in \mathbb{N}_{0}$ ), the image of $R x^{n} \otimes_{R} \Gamma_{x}(H)$ is mapped into $\Gamma_{x}(H)$. Therefore there exists $c \in R^{\circ}$ such that $c x^{n} h \in \Gamma_{x}(H)$ for all $n \gg 0$, that is, such that $c x^{n}\left(h+\Gamma_{x}(H)\right)=0$ in $G$ for all $n \gg 0$.

(iii) $\Rightarrow$ (iv) Suppose that there exist $c \in R^{\circ}$ and $n_{0} \in \mathbb{N}_{0}$ such that

$$
c x^{n}\left(h+\Gamma_{x}(H)\right)=0
$$

in $G$ for all $n \geq n_{0}$. Then, for all $j \in \mathbb{N}_{0}$, we have

$$
x^{n_{0}} c x^{j}\left(h+\Gamma_{x}(H)\right)=c^{p^{n_{0}}} x^{n_{0}+j}\left(h+\Gamma_{x}(H)\right)=0 \quad \text { in } G .
$$

Since $G$ is $x$-torsion-free, we see that $c x^{j}\left(h+\Gamma_{x}(H)\right)=0$ for all $j \geq 0$.

(iv) $\Rightarrow$ (i) Suppose that there exists $c \in R^{\circ}$ such that $c x^{n}\left(h+\Gamma_{x}(H)\right)=0$ in $G$ for all $n \geq 0$. Then $c x^{n} h \in \Gamma_{x}(H)$ for all $n \geq 0$, so that $x^{m_{0}} c x^{n} h=0$ for all $n \geq 0$. This implies that $c^{p^{m_{0}}} x^{m_{0}+n} h=0$ for all $n \geq 0$, so that $h \in 0_{H}^{*}$ by Remark 4.2(iv).

4.4. Definition. The weak parameter test ideal $\sigma^{\prime}(R)$ of $R$ is defined to be the ideal generated by 0 and all weak parameter test elements for $R$. (By a 'weak parameter test element' for $R$ we mean a $p^{w_{0}}$-weak parameter test element for $R$ for some $w_{0} \in \mathbb{N}_{0}$.)

It is easy to see that each element of $\sigma^{\prime}(R) \cap R^{\circ}$ is a weak parameter test element for $R$.

The next theorem is one of the main results of this paper. 
4.5. Theorem. Let $(R, \mathfrak{m})$ (as in Notation 1.1) be a Cohen-Macaulay local ring of dimension $d>0$. Set $H:=H_{\mathfrak{m}}^{d}(R)$, a left $R[x, f]$-module which is Artinian as an $R$-module; let $m_{0}$ be its HSL-number (see Definition 3.14), and let $q_{0}:=p^{m_{0}}$.

Set $G:=H / \Gamma_{x}(H)$, an $x$-torsion-free left $R[x, f]$-module. By Corollary 3.11 and Theorem 3.12, there exists a (uniquely determined) smallest ideal $\mathfrak{b}$ of height at least 1 in the set $\mathcal{I}(G)$ of $G$-special R-ideals.

Let $c$ be any element of $\mathfrak{b} \cap R^{\circ}$. Then $c^{q_{0}}$ is a $q_{0}$-weak parameter test element for $R$. In particular, $R$ has a $q_{0}$-weak parameter test element. In fact, the weak parameter test ideal $\sigma^{\prime}(R)$ of $R$ satisfies $\mathfrak{b}^{\left[q_{0}\right]} \subseteq \sigma^{\prime}(R) \subseteq \mathfrak{b}$.

Note. It should be noted that, in Theorem4.5 it is not assumed that $R$ is excellent. There are examples of Gorenstein local rings of characteristic $p$ which are not excellent; see [14, p. 260].

Proof. We have to show that, for an arbitrary parameter ideal $\mathfrak{a}$ of $R$ and $r \in \mathfrak{a}^{*}$, we have $c^{q_{0}} r^{p^{n}} \in \mathfrak{a}^{\left[p^{n}\right]}$ for all $n \geq m_{0}$. In the first part of the proof, we establish this in the case where $\mathfrak{a}$ is an ideal $\mathfrak{q}$ generated by a full system of parameters $a_{1}, \ldots, a_{d}$ for $R$.

Let $r \in \mathfrak{q}^{*}$, so that there exists $\widetilde{c} \in R^{\circ}$ such that $\widetilde{c} r^{p^{n}} \in \mathfrak{q}^{\left[p^{n}\right]}$ for all $n \gg$ 0 . Use $a_{1}, \ldots, a_{d}$ in the notation of Reminder 4.1(i) for $H_{\mathfrak{m}}^{d}(R)=H$, and write $a:=a_{1} \ldots a_{d}$. We have $\widetilde{c} x^{n}[r / a]=\left[\widetilde{c} r^{p^{n}} / a^{p^{n}}\right]=0$ in $H$ for all $n \gg 0$. Set $h:=[r / a] \in H$. Thus $\widetilde{c} x^{n} h=0$ for all $n \gg 0$.

It therefore follows from Corollary 3.16 that $h$ is annihilated by $\bigoplus_{n \geq m_{0}} \mathfrak{b}^{\left[p^{m_{0}}\right]} x^{n}$, so that, in particular, $c^{p^{m} 0} x^{n} h=0$ for all $n \geq m_{0}$. Hence, in $H$,

$$
\left[\frac{c^{q_{0}} r^{p^{n}}}{\left(a_{1} \ldots a_{d}\right)^{p^{n}}}\right]=c^{p^{m_{0}}} x^{n}\left[\frac{r}{a_{1} \ldots a_{d}}\right]=c^{p^{m_{0}}} x^{n} h=0 \quad \text { for all } n \geq m_{0} .
$$

Since $R$ is Cohen-Macaulay, we can now deduce from Reminder 4.1(i) that $c^{q_{0}} r^{p^{n}} \in$ $\mathfrak{q}^{\left[p^{n}\right]}$ for all $n \geq m_{0}$, as required (for $\mathfrak{q}$ ).

Now let $\mathfrak{a}$ be an arbitrary parameter ideal of $R$. A proper ideal in a CohenMacaulay local ring is a parameter ideal if and only if it can be generated by part of a system of parameters. In view of the first part of this proof, we can, and do, assume that ht $\mathfrak{a}<d$. There exist a system of parameters $a_{1}, \ldots, a_{d}$ for $R$ and an integer $i \in\{0, \ldots, d-1\}$ such that $\mathfrak{a}=\left(a_{1}, \ldots, a_{i}\right) R$. Let $r \in \mathfrak{a}^{*}$. Then, for each $v \in \mathbb{N}$, we have $r \in\left(\left(a_{1}, \ldots, a_{i}, a_{i+1}^{v}, \ldots, a_{d}^{v}\right) R\right)^{*}$, and, since $a_{1}, \ldots, a_{i}, a_{i+1}^{v}, \ldots, a_{d}^{v}$ is a system of parameters for $R$, it follows from the first part of this proof that

$$
\begin{aligned}
c^{q_{0}} r^{p^{n}} & \in\left(\left(a_{1}, \ldots, a_{i}, a_{i+1}^{v}, \ldots, a_{d}^{v}\right) R\right)^{\left[p^{n}\right]} \\
& =\left(a_{1}^{p^{n}}, \ldots, a_{i}^{p^{n}}, a_{i+1}^{v p^{n}}, \ldots, a_{d}^{v p^{n}}\right) R \quad \text { for all } n \geq m_{0} .
\end{aligned}
$$

Therefore, for all $n \geq m_{0}$,

$$
c^{q_{0}} r^{p^{n}} \in \bigcap_{v \in \mathbb{N}}\left(a_{1}^{p^{n}}, \ldots, a_{i}^{p^{n}}, a_{i+1}^{v p^{n}}, \ldots, a_{d}^{v p^{n}}\right) R \subseteq \bigcap_{v \in \mathbb{N}}\left(\mathfrak{a}^{\left[p^{n}\right]}+\mathfrak{m}^{v p^{n}}\right)=\mathfrak{a}^{\left[p^{n}\right]}
$$

by Krull's Intersection Theorem. This shows that $c^{q_{0}}$ is a $q_{0}$-weak parameter test element for $R$, so that, since $\mathfrak{b}$ can be generated by elements in $\mathfrak{b} \cap R^{\circ}$, it follows that $\mathfrak{b}^{\left[q_{0}\right]} \subseteq \sigma^{\prime}(R)$.

Now let $c \in \sigma^{\prime}(R) \cap R^{\circ}$; we suppose that $c \notin \mathfrak{b}$ and seek a contradiction. Thus $\mathfrak{b} \subset \mathfrak{b}+R c$. Let $L:=\operatorname{ann}_{G}(\mathfrak{b} R[x, f])$ and $L^{\prime}:=\operatorname{ann}_{G}((\mathfrak{b}+R c) R[x, f])$, two special annihilator submodules of the $x$-torsion-free left $R[x, f]$-module $G$. Since $L$ 
corresponds to the $G$-special $R$-ideal $\mathfrak{b}$, we must have $L^{\prime} \subset L$, since otherwise we would have

$$
(\mathfrak{b}+R c) R[x, f] \subseteq \text { gr-ann }_{R[x, f]} L^{\prime}=\operatorname{gr}^{-a n n_{R[x, f]}} L=\mathfrak{b} R[x, f] .
$$

Therefore there exists $h \in H$ such that $h+\Gamma_{x}(H)$ is annihilated by $\mathfrak{b} R[x, f]$ but not by $(\mathfrak{b}+R c) R[x, f]$. Since ht $\mathfrak{b} \geq 1$, it follows from Lemma 4.3 that $h \in 0_{H}^{*}$.

Choose a system of parameters $a_{1}, \ldots, a_{d}$ for $R$; use $a_{1}, \ldots, a_{d}$ in the notation of Reminder 4.1(i) for $H_{\mathfrak{m}}^{d}(R)=H$, and write $a:=a_{1} \ldots a_{d}$. There exist $r \in R$ and $j \in \mathbb{N}_{0}$ such that $h=\left[r / a^{j}\right]$. By Remark 4.2(vi), we have $r \in\left(\left(a_{1}^{j}, \ldots, a_{d}^{j}\right) R\right)^{*}$. Since $c$ is a weak parameter test element for $R$, we see that $c r^{p^{n}} \in\left(a_{1}^{j p^{n}}, \ldots, a_{d}^{j p^{n}}\right) R$ for all $n \gg 0$, so that $c x^{n} h=0$ for all $n \gg 0$. Thus there is some $n_{0} \in \mathbb{N}_{0}$ such that $c x^{n}\left(h+\Gamma_{x}(H)\right)=0$ in $G$ for all $n \geq n_{0}$. Therefore, for all $j \in \mathbb{N}_{0}$, we have

$$
x^{n_{0}} c x^{j}\left(h+\Gamma_{x}(H)\right)=c^{p^{n_{0}}} x^{n_{0}+j}\left(h+\Gamma_{x}(H)\right)=0,
$$

so that $c x^{j}\left(h+\Gamma_{x}(H)\right)=0$ because $G$ is $x$-torsion-free. Thus $h+\Gamma_{x}(H)$ is annihilated by $R c R[x, f]$ as well as by $\mathfrak{b} R[x, f]$. This is a contradiction.

Therefore $\mathfrak{b}^{\left[q_{0}\right]} \subseteq \sigma^{\prime}(R) \subseteq \mathfrak{b}$, since $\sigma^{\prime}(R)$ can be generated by its elements that lie in $R^{\circ}$.

Use $R^{\prime}$ to denote $R$ (as in Notation 1.1) regarded as an $R$-module by means of $f$. With this notation, $f: R \longrightarrow R^{\prime}$ becomes a homomorphism of $R$-modules. Recall that, when $(R, \mathfrak{m})$ is a local ring of dimension $d>0$, we say that $R$ is $F$ injective precisely when the induced homomorphisms $H_{\mathfrak{m}}^{i}(f): H_{\mathfrak{m}}^{i}(R) \longrightarrow H_{\mathfrak{m}}^{i}\left(R^{\prime}\right)$ are injective for all $i=0, \ldots, d$. See R. Fedder and K-i. Watanabe [5, Definition 1.7] and the ensuing discussion.

4.6. Corollary. Let $(R, \mathfrak{m})$ be an $F$-injective Cohen-Macaulay local ring of dimension $d>0$. The left $R[x, f]$-module $H:=H_{\mathfrak{m}}^{d}(R)$ is $x$-torsion-free. By Theorem 3.12, there exists a (uniquely determined) smallest ideal $\mathfrak{b}$ of height at least 1 in the set $\mathcal{I}(H)$ of $H$-special $R$-ideals.

Let $c$ be any element of $\mathfrak{b} \cap R^{\circ}$. Then $c$ is a parameter test element for $R$. In fact, $\mathfrak{b}$ is the parameter test ideal of $R$ (see [18, Definition 4.3]).

Note. It should be noted that, in Corollary 4.6, it is not assumed that $R$ is excellent.

Proof. With the notation of Theorem 4.5 the HSL-number $m_{0}$ of $H$ is 0 when $R$ is $F$-injective, and so $q_{0}=1$ and $G \cong H$ in this case. By Theorem 4.5 each element $c \in \mathfrak{b} \cap R^{\circ}$ is a $q_{0}$-weak parameter test element for $R$, that is, a parameter test element for $R$. Since $R$ has a parameter test element, its parameter test ideal $\sigma(R)$ is equal to the ideal of $R$ generated by all parameter test elements. By Theorem 4.5. we therefore have $\mathfrak{b}=\mathfrak{b}^{\left[q_{0}\right]} \subseteq \sigma(R) \subseteq \sigma^{\prime}(R) \subseteq \mathfrak{b}$.

4.7. Corollary. Let $(R, \mathfrak{m})$ be an $F$-injective Gorenstein local ring of dimension $d>0$. The left $R[x, f]$-module $H:=H_{\mathfrak{m}}^{d}(R)$ is $x$-torsion-free. By Theorem 3.12 , there exists a (uniquely determined) smallest ideal $\mathfrak{b}$ of height at least 1 in the set $\mathcal{I}(H)$ of $H$-special $R$-ideals.

Let $c$ be any element of $\mathfrak{b} \cap R^{\circ}$. Then $c$ is a test element for $R$. In fact, $\mathfrak{b}$ is the test ideal of $R$.

Note. It should be noted that, in Corollary 4.7, it is not assumed that $R$ is excellent. 
Proof. This follows immediately from Corollary 4.6 once it is recalled that an $F$ injective Cohen-Macaulay local ring is reduced and that a parameter test element for a reduced Gorenstein local ring $R$ of characteristic $p$ is automatically a test element for $R$; see the proof of [11, Proposition 4.1].

\section{Special $R$-IDEAls And Enescu's $F$-StABle PRIMES}

The purpose of this section is to establish connections between the work in $₫ 3$ and 94 above and F. Enescu's $F$-stable primes of an $F$-injective Cohen-Macaulay local ring $(R, \mathfrak{m})$, defined in $[3, \S 2]$.

5.1. Notation. Throughout this section, $(R, \mathfrak{m})$ will be assumed to be a CohenMacaulay local ring of dimension $d>0$, and we shall let $a_{1}, \ldots, a_{d}$ denote a fixed system of parameters for $R$, and set $\mathfrak{q}:=\left(a_{1}, \ldots, a_{d}\right) R$. We shall use $a_{1}, \ldots, a_{d}$ in the notation of Reminder 4.1(i) for $H:=H_{\mathfrak{m}}^{d}(R)$, and write $a:=a_{1} \ldots a_{d}$.

For each $b \in R$, we define (following Enescu [3, Definition 1.1]) the ideal $\mathfrak{q}(b)$ by

$$
\mathfrak{q}(b):=\left\{c \in R: c b^{p^{n}} \in \mathfrak{q}^{\left[p^{n}\right]} \text { for all } n \gg 0\right\} .
$$

(Actually, Enescu only made this definition when $b \notin \mathfrak{q}$; however, the right-hand side of the above display is equal to $R$ when $b \in \mathfrak{q}$, and there is no harm in our defining $\mathfrak{q}(b)$ to be $R$ in this case.) In view of Reminder 4.1(i), the ideal $\mathfrak{q}(b)$ is equal to the ultimate constant value of the ascending chain $\left(\mathfrak{b}_{n}\right)_{n \in \mathbb{N}_{0}}$ of ideals of $R$ for which $\bigoplus_{n \in \mathbb{N}_{0}} \mathfrak{b}_{n} x^{n}=\operatorname{gr}^{-a n n n_{R[x, f]}} R[x, f][b / a]$, the graded annihilator of the $R[x, f]$-submodule of $H$ generated by $[b / a]$.

Now consider the special case in which $R$ is (also) $F$-injective. Then the left $R[x, f]$-module $H$ is $x$-torsion-free, and so it follows from Lemma 1.9 that, for each

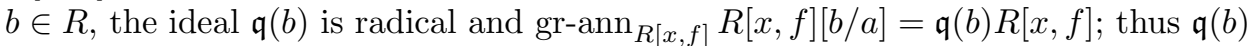
is an $H$-special $R$-ideal. We again follow Enescu and set

$$
Z_{\mathfrak{q}, R}:=\{\mathfrak{q}(b): b \in R \backslash \mathfrak{q}\} .
$$

Enescu proved, in [3, Theorem 2.1], that (when $(R, \mathfrak{m})$ is Cohen-Macaulay and $F$-injective) the set of maximal members of $Z_{\mathfrak{q}, R}$ is independent of the choice of $\mathfrak{q}$, is finite, and consists of prime ideals. The next theorem shows that the set of maximal members of $Z_{\mathfrak{q}, R}$ is actually equal to the set of maximal members of $\mathcal{I}(H) \backslash\{R\}$ : we saw in Lemma 3.8 that this set consists of prime ideals, and in Corollary 3.11 that it is finite.

5.2. Theorem. Let the situation and notation be as in Notation [5.1, and suppose that the Cohen-Macaulay local ring $(R, \mathfrak{m})$ is $F$-injective. Then the set of maximal members of $Z_{\mathfrak{q}, R}$ is equal to the set of maximal members of $\mathcal{I}(H) \backslash\{R\}$.

Proof. The comments in Notation 5.1 show that $Z_{\mathfrak{q}, R} \subseteq \mathcal{I}(H)$; clearly, no member of $Z_{\mathfrak{q}, R}$ can be equal to $R$. It is therefore sufficient for us to show that a maximal member $\mathfrak{p}$ of $\mathcal{I}(H) \backslash\{R\}$ must belong to $Z_{\mathfrak{q}, R}$.

Let $L \in \mathcal{A}(H)$ be the special annihilator submodule of $H$ corresponding to $\mathfrak{p}$. Now $H$ is an Artinian $R$-module: let $h$ be a non-zero element of the socle of $L$. By Lemma 3.8, we have gr-ann $\operatorname{and}_{[x, f]} R[x, f] h=\mathfrak{p} R[x, f]$. However, for each $j \in \mathbb{N}$, we have $R\left[1 / a^{j}\right] \cong R /\left(a_{1}^{j}, \ldots, a_{d}^{j}\right) R$, by Remark 4.2(vii), so that

$$
\operatorname{Hom}_{R}\left(R / \mathfrak{m}, R\left[1 / a^{j}\right]\right) \cong \operatorname{Hom}_{R}\left(R / \mathfrak{m}, R /\left(a_{1}^{j}, \ldots, a_{d}^{j}\right) R\right) \cong \operatorname{Ext}_{R}^{d}(R / \mathfrak{m}, R)
$$


by [2, Lemma 1.2.4]. It follows that it is possible to write $h$ in the form $h=[r / a]$ for some $r \in R$, and therefore $\mathfrak{p}=\mathfrak{q}(r) \in Z_{\mathfrak{q}, R}$.

\section{REFERENCES}

1. M. P. Brodmann and R. Y. Sharp, Local cohomology: an algebraic introduction with geometric applications, Cambridge Studies in Advanced Mathematics 60, Cambridge University Press, 1998. MR:1613627 (99h:13020)

2. W. Bruns and J. Herzog, Cohen-Macaulay rings, Cambridge Studies in Advanced Mathematics 39, Cambridge University Press, 1993. MR1251956 (95h:13020)

3. F. Enescu, F-injective rings and F-stable primes, Proc. Amer. Math. Soc. 131 (2003) 33793386. MR 1990626 (2004f:13003)

4. R. Fedder, F-purity and rational singularity in graded complete intersection rings, Transactions Amer. Math. Soc. 301 (1987) 47-62. MR0879562 (88h:14002)

5. R. Fedder and K-i. Watanabe, A characterization of F-regularity in terms of F-purity, in: M. Hochster, C. Huneke and J. D. Sally (Eds.), Commutative algebra: proceedings of a microprogram held June 15 - July 2, 1987, Mathematical Sciences Research Institute Publications 15, Springer, New York, 1989, pp. 227-245. MR1015520(91k:13009)

6. N. Hara, K-i. Watanabe, The injectivity of Frobenius acting on cohomology and local cohomology modules, Manuscripta Math. 90 (1996) 301-315. MR.1397659 (97i:13016)

7. R. Hartshorne and R. Speiser, Local cohomological dimension in characteristic p, Annals of Math. 105 (1977) 45-79. MR0441962 (56:353)

8. M. Hochster and C. Huneke, Tight closure, invariant theory and the Briançon-Skoda Theorem, J. Amer. Math. Soc. 3 (1990) 31-116. MR1017784 (91g:13010)

9. M. Hochster and C. Huneke, F-regularity, test elements, and smooth base change, Transactions Amer. Math. Soc. 346 (1994) 1-62. MR1273534 (95d:13007)

10. C. Huneke, Tight closure and its applications, Conference Board of the Mathematical Sciences Regional Conference Series in Mathematics 88, American Mathematical Society, Providence, 1996. MR $1377268(96 \mathrm{~m}: 13001)$

11. C. Huneke, Tight closure, parameter ideals and geometry, in: J. Elias, J. M. Giral, R. M. MiróRoig and S. Zarzuela (Eds.), Six lectures on commutative algebra, Progress in Mathematics 166, Birkhäuser, Basel, 1998, pp. 187-239. MR1648666 (99j:13001)

12. M. Katzman and R. Y. Sharp, Uniform behaviour of the Frobenius closures of ideals generated by regular sequences, J. Algebra, 295 (2006) 231-246. MR2188859(2006i:13007)

13. G. Lyubeznik, F-modules: applications to local cohomology and D-modules in characteristic $p>0$, J. reine angew. Math. 491 (1997) 65-130. MR.1476089 (99c:13005)

14. H. Matsumura, Commutative algebra: second edition, Benjamin/Cummings, Reading, Massachusetts, 1980. MR0575344(82i:13003)

15. L. O'Carroll, On the generalized fractions of Sharp and Zakeri, J. London Math. Soc. (2) 28 (1983) 417-427. MR0724710 (85e:13025)

16. R. Y. Sharp and N. Nossem, Ideals in a perfect closure, linear growth of primary decompositions, and tight closure, Transactions Amer. Math. Soc. 356 (2004) 3687-3720. MR2055750 (2005a:13009)

17. K. E. Smith, Tight closure of parameter ideals, Inventiones mathematicae 115 (1994) 41-60. MR.1248078 (94k:13006)

18. K. E. Smith, Test ideals in local rings, Transactions Amer. Math. Soc. 347 (1995) 3453-3472. MR:1311917 (96c:13008)

19. Y. Yoshino, Skew-polynomial rings of Frobenius type and the theory of tight closure, Communications in Algebra 22 (1994) 2473-2502. MR1271618 (95h:16037)

Department of Pure Mathematics, University of Sheffield, Hicks Building, Sheffield S3 7RH, United Kingdom

E-mail address: R.Y.Sharp@sheffield.ac.uk 